Citation: Hirt C (2012) Efficient and accurate high-degree spherical harmonic synthesis of gravity field functionals at the Earth's surface using the gradient approach. Journal of Geodesy, 86(9), 729-744, DOI: 10.1007/s00190-012-0550-y.

\title{
Efficient and accurate high-degree spherical harmonic synthesis of gravity field functionals at the Earth's surface using the gradient approach
}

\section{Christian Hirt}

Western Australian Centre for Geodesy \& The Institute for Geoscience Research, Curtin University of Technology, GPO Box U1987, Perth, WA 6845, Australia

Email: c.hirt@curtin.edu.au

\begin{abstract}
Spherical harmonic synthesis (SHS) of gravity field functionals at the Earth's surface requires the use of heights. The present study investigates the gradient approach as an efficient yet accurate strategy to incorporate height information in SHS at densely-spaced multiple points. Taylor series expansions of commonly used functionals quasigeoid heights, gravity disturbances and vertical deflections are formulated, and expressions of their radial derivatives are presented to arbitrary order. Numerical tests show that first-order gradients, as introduced by Rapp (J Geod 71(5): 282-289, 1997) for degree-360 models, produce cm- to dm-level RMS approximation errors over rugged terrain when applied with EGM2008 to degree 2190. Instead, higher-order Taylor expansions are recommended that are capable of reducing approximation errors to insignificance for practical applications. Because the height information is separated from the actual synthesis, the gradient approach can be applied along with existing highly-efficient SHS routines to compute surface functionals at arbitrarily dense grid points. This confers considerable computational savings (above or well above one order of magnitude) over conventional point-by-point SHS. As an application example, an ultrahigh resolution model of surface gravity functionals (EurAlpGM2011) is constructed over the entire European Alps that incorporates height information in the SHS at 12,000,000 surface points. Based on EGM2008 and residual topography data, quasigeoid heights, gravity disturbances and vertical deflections are estimated at $\sim 200 \mathrm{~m}$ resolution. As a conclusion, the gradient approach is efficient and accurate for high-degree SHS at multiple points at the Earth's surface.
\end{abstract}

\section{Keywords}

Spherical harmonic synthesis, quasigeoid, gravity disturbances, vertical deflections, radial derivatives 


\section{Introduction}

Spherical harmonic (SH) expansions are widely used to describe the gravitational potential and disturbing potential of Earth (e.g., Torge 2001) and of other celestial objects such as the Moon, and the terrestrial planets (e.g., Wieczorek 2007). Over many years, both efficient and stable algorithms for spherical harmonic synthesis (SHS), i.e., the computation of gravity field functionals from SH coefficients, have been devised or investigated (e.g., Rizos 1979; Tscherning and Poder 1982; Tscherning et al. 1983; Wenzel 1985; Abd-Elmotaal 1997; Wenzel 1999; Holmes and Featherstone 2002a, 2002b, 2002c, Holmes 2003; Bethencourt et al. 2005; Casotto and Fantino 2007), and high-degree Earth global gravitational models (GGMs) were developed. Examples are EIGEN-6 (Förste et al. 2011) to degree 1420, EGM2008 (Pavlis et al. 2008) to 2190, GPM98A and GPM98B (Wenzel 1998) to 1800. Algorithms capable of extending SH expansions beyond or well beyond degree 2190 are now emerging, which is seen by recent studies of e.g., Fukushima (2011); Gruber et al. (2011a); Šprlák (2011), Wittwer et al. (2008) and Jekeli et al. (2007).

For efficient high-degree SHS of gravity field quantities at regularly-spaced grid points at some reference ellipsoid or sphere, some of the algorithms recently proposed (e.g., Fukushima 2011) or routinely used in practice (e.g., Holmes and Pavlis 2008) use the numerically efficient expression of the disturbing potential $T$ (cf. Holmes and Featherstone 2002a, c)

$T(\varphi, \lambda, r)=\frac{G M}{r} \sum_{m=0}^{M} c_{m} \cos m \lambda+s_{m} \sin m \lambda$

with the lumped coefficients

$$
\begin{aligned}
& C_{m}=\sum_{n=\max (2, m)}^{M}\left(\frac{a}{r}\right)^{n} \bar{C}_{n m} \bar{P}_{n m}(\sin \varphi) \\
& S_{m}=\sum_{n=\max (2, m)}^{M}\left(\frac{a}{r}\right)^{n} \bar{S}_{n m} \bar{P}_{n m}(\sin \varphi)
\end{aligned}
$$

where $M$ is the maximum degree, $G M$ and $a$ are the GGM-specific scaling parameters, the spherical coordinates latitude $\varphi$, longitude $\lambda$ and geocentric radius $r$ specify the computation point, $\bar{P}_{n m}(\sin \varphi)$ are the fully-normalized Associated Legendre Functions of degree $n$ and order $m$, and $\bar{C}_{n m} \bar{S}_{n m}$ are the fully-normalized SH coefficients referred to some normal gravity field. Because the $c_{m}, s_{m}$ are independent of $\lambda$, Eq. (2) needs to be evaluated only once for computation points densely spaced along a parallel (i.e., $\varphi$ constant) if the geocentric radius $r$ is constant as well (e.g., Tscherning and Poder 1982; Holmes and Featherstone 2002a), and the efficiency can be further increased for points equally spaced in longitude (see Rizos 1979; Abd-Elmotaal 1997 for details).

1.1 The height problem of SHS 
Due to spatial resolution conferred by high-degree GGMs such as EGM2008, SHS of GGM functionals at the Earth's surface is an application of increasing importance. Examples of such surface functionals are (Molodensky) quasigeoid heights and gravity disturbances (cf. Torge 2001), and Helmert and Molodensky vertical deflections (see Jekeli 1999). Importantly, SHS at the Earth's surface requires the 3D location $\left(\varphi, \lambda, r_{P}\right)$ of the computation point (cf. Rapp 1997, p. 283; Claessens et al. 2009, p. 223; Hirt et al. 2010a, p. 563; Hirt 2011; ibid Section 4), which can be sourced, e.g., from elevation models.

The geocentric radius $r_{P}$ of the computation point (situated at the Earth's surface) enters the SHS expansions both via the $G M / r_{P}$ scale factor and the $\left(a / r_{P}\right)^{n}$ attenuation factor. Unfortunately, over most land areas, Earth's topography causes $r_{P}$ to be a highly-varying quantity along geodetic parallels. As a consequence, computation of GGM functionals at the Earth's topography - even at regularly-spaced grid points - requires evaluation of the SH expansions [Eqs. (1), (2)] separately for each $\left(\varphi, \lambda, r_{P}\right)$ triplet (e.g., Holmes 2003, p. 25). For multiple grid points, this is a very time-consuming operation in practice (Holmes 2003, p. 129f; Claessens et al. 2009, p. 223).

A pragmatic solution to this "height problem of high-degree SHS" lies in the use of Taylor series expansions to continue GGM functionals from some reference surface to the topography. The required functionals and radial derivatives can be efficiently computed with accelerated SHS routines. This idea goes back to at least Rapp (1997, p. 283) who uses firstorder gradients to compute quasigeoid heights at the Earth's surface. Later, in the context of synthetic [simulated] gravity field modelling, Holmes (2003) makes extensive use of firstorder Taylor expansions to continue functionals over short vertical distances, say $\sim 100 \mathrm{~m}$, from the ellipsoid to the geoid. Tóth (2005) and Keller and Sharifi (2005) use higher-order gradients in the context of satellite gradiometry, and Fantino and Casotto (2009) derived firstto third-order gradients of the gravitational potential. However, to the knowledge of the author, the use of higher-order gradients has not yet been systematically presented, investigated and applied for the accurate continuation of high-degree GGM functionals, from the ellipsoid to Earth's surface.

\subsection{Aim of this study}

The aim of this study is to explore the gradient approach as a viable and pragmatic solution to the high-degree SHS height problem. The approach presented here is an extension of Rapp's (1997) method to compute quasigeoid heights at the Earth's surface from first-order Taylor expansions, and Holmes's (2003) simulated modelling over short vertical distances. We use elevation data along with third-order Taylor expansions to obtain grids of quasigeoid heights at the Earth's surface (Section 2). This requires first- to third-order radial derivatives of the quasigeoid, which are synthesised at some constant height above the reference ellipsoid using existing numerically-efficient algorithms. Section 3 then extends the gradient approach to gravity disturbances and vertical deflections, and gives the radial derivatives in $\mathrm{SH}$ representation. Numerical tests in Sections 2 and 3 demonstrate that the use of some constant 
average reference height in the SHS is beneficial to reduce the approximation errors of the Taylor expansions to insignificance for degree-2190 SHS even over the most rugged regions of Earth.

Finally, an application example for the gradient approach is given in Section 4, demonstrating the capability of the method to incorporate high-resolution elevation data in high-degree SHS. At 12 million points, we compute EGM2008 quasigeoid heights, gravity disturbances and vertical deflections at the Earth's surface over the entire European Alps, and use beyond the EGM2008 resolution topography-implied gravity-effects as high-frequency augmentation. This yields accurate gravity field functionals at densely-spaced 3D surface points (Section 4).

\section{The gradient approach for height anomalies $\zeta$}

Here and in the following sections, we denote the geodetic coordinates of a point $P$ with $\phi$ (geodetic latitude), $\lambda$ (longitude) and $h$ (ellipsoidal height). Spherical (polar) coordinates which are required for the evaluation of $\mathrm{SH}$ expansions are denoted with $\varphi$ (geocentric latitude), $\lambda$ (longitude) and $r$ (geocentric radius). The transformation from geodetic to spherical coordinates via global 3D Cartesian coordinates is described e.g., in Wenzel (1985 p. 130f), Jekeli (2006 section 2.1.5 ibid) and Torge (2001, chapter 4 ibid). We further assume throughout the paper that the zonal harmonics of some normal gravity field have been removed from the $\bar{C}_{n m}$ coefficients (see, e.g., Smith 1998 for details).

\subsection{Rapp’s approach}

Rapp (1997) describes a formalism to calculate geoid undulations $N$ (aka geoid heights) via quasigeoid heights $\zeta$ (aka height anomalies) from the disturbing potential $T$. Based on Heiskanen and Moritz (1967), Rapp (1997, p. 282) obtains $\zeta$ at 3D-locations $(\varphi, \lambda, r)$ from

$$
\begin{aligned}
& \zeta(\varphi, \lambda, r)=\frac{T(\varphi, \lambda, r)}{\gamma}=\frac{G M}{\gamma r} \sum_{m=0}^{M}\left[\cos m \lambda \sum_{n=\mu}^{M}\left(\frac{a}{r}\right)^{n} \bar{C}_{n m} \bar{P}_{n m}(\sin \varphi)+\right. \\
& \left.\sin m \lambda \sum_{n=\mu}^{M}\left(\frac{a}{r}\right)^{n} \bar{S}_{n m} \bar{P}_{n m}(\sin \varphi)\right]
\end{aligned}
$$

where $n=\mu=\max (2, m), \gamma$ is the normal gravity at $(\varphi, \lambda, r)$, and the summation order of $n$ and $m$ has been modified to allow for efficient SHS (see above). Rapp (1997) states that $\zeta$ is dependent on $r_{P}$, which is the geocentric distance of the computation point $P$ located at the Earth’s topography or above. He then approximates $\zeta_{P}$ using a first-order Taylor expansion

$$
\zeta_{P}^{\text {Rapp }}\left(\varphi, \lambda, r_{P}\right) \approx \zeta_{0}\left(\varphi, \lambda, r_{E}\right)+C_{1 \mathrm{~A}}(\varphi, \lambda, h)+C_{1 \mathrm{~B}}(\varphi, \lambda, h)
$$

where $\zeta_{0}$ the quasigeoid height at $\left(\varphi, \lambda, r_{E}\right)$ and $r_{E}$ is the ellipsoidal radius (e.g., Claessens 2006 p. 18ff), i.e., the geocentric distance to the point $P_{0}$ on the ellipsoid. Note that Rapp's 
C1-term is split here into two components $C_{1 \mathrm{~A}}$ and $C_{1 \mathrm{~B}}$. For multiple points arranged along geodetic parallels, $r_{E}$ and $\varphi$ are constant, which allows $\zeta_{0}$ to be evaluated at the ellipsoid $\left(\varphi, \lambda, r_{E}\right)$ using the accelerated SHS routines (see Section 1$)$. To the understanding of the author, this is described by Rapp (1997, p. 283) as "computer efficiency". Rapp writes the first-order Taylor term

$$
C_{1 \mathrm{~A}}(\varphi, \lambda, h)=\left.\frac{\partial \zeta}{\partial r}\right|_{r_{E}} h
$$

as a function of the radial derivative $\partial \zeta / \partial r$ [Eq. (10)] and $h$ (ellipsoidal height of $P$ ). Again, using a constant $r_{E}$ allows accelerated SHS of the $C_{1 \mathrm{~A}}$-term. Rapp further includes a term to model the change of $\zeta$ with the height-dependent $\gamma$

$$
C_{1 \mathrm{~B}}(\varphi, \lambda, h)=\left.\frac{\partial \zeta}{\partial \gamma} \frac{\partial \gamma}{\partial h}\right|_{r_{E}} h
$$

where $\partial \zeta / \partial \gamma=-T(\varphi, \lambda, r) / \gamma^{2} \approx-\zeta_{0} / \gamma$ and $\partial \gamma / \partial h$ is the normal gravity gradient $\left(\approx-3.086 \mu \mathrm{ms}^{-2} / \mathrm{m}\right.$, Torge 2001, p. 111). Rapp approximates the ellipsoidal height $h$ in Eqs. (5) and (6) with the orthometric height $H$, and, finally, approximates geoid heights

$$
N(\varphi, \lambda) \approx \zeta_{P}^{\text {Rapp }}\left(\varphi, \lambda, r_{P}\right)+C_{2}(\varphi, \lambda, H)
$$

by accounting for the geoid-to-quasigeoid separation term

$$
C_{2}(\varphi, \lambda, H)=\frac{\Delta g_{B}}{\bar{\gamma}} H
$$

that is a function of the Bouguer-anomaly $\Delta g_{B}$, orthometric height $H$ and a mean normal gravity value $\bar{\gamma}$ (Torge 2001, p. 292). The geoid-quasigeoid separation term has been much discussed in the literature (e.g., Featherstone and Kirby 1998; Nahavandchi 2002; Ågren 2004) and refined (e.g., Sjöberg 2006; Tenzer et al. 2006; Flury and Rummel 2009; Sjöberg 2010), while less attention has been paid to the first-order Taylor series [Eq. (4) and (5)] in the context of high-degree SHS. Here we do not further deal with the geoid-quasigeoid separation [Eq. (8)], but focus on a refinement of Rapp's quasigeoid approximation at the Earth's surface. We note that Rapp (1997) investigated the formalism [Eqs. (3) to (8)] for degree-360 and not for degree-2190 expansions, that were not yet available at that time. As will be shown here, high-degree SHS requires extension of Rapp's approach (Sections 2.2, 2.3) to diminish $\zeta$-approximation errors over mountain terrain (Section 2.4).

\subsection{Introducing higher degree terms}


As a first refinement, we extend Rapp's (1997) first-order Taylor series to third-order while retaining the $C_{1 \mathrm{~B}}$-term. Extended to third-order, the expression for $\zeta_{P}$ reads:

$$
\zeta_{P}\left(\varphi, \lambda, r_{P}\right)=\underbrace{\zeta_{0}\left(\varphi, \lambda, r_{E}\right)}_{\begin{array}{c}
\text { 0th-order } \\
\text { contribution }
\end{array}}+\underbrace{\left.\frac{\partial \zeta}{\partial r}\right|_{r_{E}}}_{\begin{array}{c}
\text { 1st-order } \\
\text { contribution }
\end{array}} h+\underbrace{\left.\frac{1}{2} \frac{\partial^{2} \zeta}{\partial r^{2}}\right|_{r_{E}}}_{\begin{array}{c}
2 \text { nd-order } \\
\text { contribution }
\end{array}} h^{2}+\underbrace{\left.\frac{1}{6} \frac{\partial^{3} \zeta}{\partial r^{3}}\right|_{r_{E}}}_{\begin{array}{c}
\text { 3rd-order } \\
\text { contribution }
\end{array}} h^{3}+C_{1 \mathrm{~B}}(\varphi, \lambda, h)
$$

where the required first- to third-order radial gradients of $\zeta$ are (see also Fantino and Casotto (2009, p. 602f)

$$
\begin{gathered}
\zeta_{r(1)}=\frac{\partial \zeta}{\partial r}=-\frac{G M}{r^{2} \gamma} \sum_{m=0}^{M}\left[\begin{array}{c}
\cos m \lambda \sum_{n=\mu}^{M}(n+1)\left(\frac{a}{r}\right)^{n} \bar{C}_{n m} \bar{P}_{n m}(\sin \varphi)+ \\
\sin m \lambda \sum_{n=\mu}^{M}(n+1)\left(\frac{a}{r}\right)^{n} \bar{S}_{n m} \bar{P}_{n m}(\sin \varphi)
\end{array}\right], \\
\zeta_{r(2)}=\frac{\partial^{2} \zeta}{\partial r^{2}}=+\frac{G M}{r^{3} \gamma} \sum_{m=0}^{M}\left[\begin{array}{r}
\cos m \lambda \sum_{n=\mu}^{M}(n+1)(n+2)\left(\frac{a}{r}\right)^{n} \bar{C}_{n m} \bar{P}_{n m}(\sin \varphi)+ \\
\sin m \lambda \sum_{n=\mu}^{M}(n+1)(n+2)\left(\frac{a}{r}\right)^{n} \bar{S}_{n m} \bar{P}_{n m}(\sin \varphi)
\end{array}\right],
\end{gathered}
$$

and

$$
\begin{aligned}
& \zeta_{r(3)}=\frac{\partial^{3} \zeta}{\partial r^{3}}=-\frac{G M}{r^{4} \gamma} \sum_{m=0}^{M}\left[\cos m \lambda \sum_{n=\mu}^{M}(n+1)(n+2)(n+3)\left(\frac{a}{r}\right)^{n} \bar{C}_{n m} \bar{P}_{n m}(\sin \varphi)+\right. \\
& \left.\sin m \lambda \sum_{n=\mu}^{M}(n+1)(n+2)(n+3)\left(\frac{a}{r}\right)^{n} \bar{S}_{n m} \bar{P}_{n m}(\sin \varphi)\right]
\end{aligned}
$$

The radial derivatives of order $k$ can be constructed from simple mathematical principles (the scale factor between subsequent orders differs by $\left(-r^{-1}\right)$ and the attenuation factor is multiplied by the $(n+k)$-factors), see also Rummel and van Gelderen (1995). This allows formulation of a compact expression for radial derivatives of arbitrary order $k$

$$
\begin{aligned}
& \zeta_{r(k)}=\frac{\partial^{k} \zeta}{\partial r^{k}}=(-1)^{k} \frac{G M}{r^{k+1} \gamma} \sum_{m=0}^{M}\left[\cos m \lambda \sum_{n=\mu}^{M}\left\{\prod_{i=1}^{k}(n+i)\right\}\left(\frac{a}{r}\right)^{n} \bar{C}_{n m} \bar{P}_{n m}(\sin \varphi)+\right. \\
& \left.\sin m \lambda \sum_{n=\mu}^{M}\left\{\prod_{i=1}^{k}(n+i)\right\}\left(\frac{a}{r}\right)^{n} \bar{S}_{n m} \bar{P}_{n m}(\sin \varphi)\right]
\end{aligned}
$$

where $\zeta_{r(k)}=\partial^{k} \zeta / \partial r^{k}$ is the shorthand for the $k$-th radial derivative of $\zeta$ that can be used to expand $\zeta_{P}$ to a maximum order $K$ : 
$\zeta_{P}\left(\varphi, \lambda, r_{P}\right)=\zeta_{0}\left(\varphi, \lambda, r_{E}\right)+\left\{\left.\sum_{k=1}^{K} \frac{1}{k !} \frac{\partial^{k} \zeta}{\partial r^{k}}\right|_{r_{E}} h^{k}\right\}+C_{1 \mathrm{~B}}(\varphi, \lambda, h)$

\subsection{Refinement through reference height $\bar{h}$}

As a second refinement to Rapp's approach, we introduce some constant ellipsoidal reference height $\bar{h}$ (e.g., average elevation of a working area) in order to shorten the vertical distances along which $\zeta$ is continued, which, in turn, reduces the approximation errors. All $h$ of the topography now refer to $\bar{h}$ and the SHS functionals are evaluated at $r_{E}+\bar{h}$, which, importantly, is constant along geodetic parallels, so allows numerically efficient SHS. The expansion for $\zeta_{P}$ reads:

$$
\zeta_{P}\left(\varphi, \lambda, r_{P}\right) \approx \zeta_{0}\left(\varphi, \lambda, r_{E}+\bar{h}\right)+\left\{\left.\sum_{k=1}^{K} \frac{1}{k !} \frac{\partial^{k} \zeta}{\partial r^{k}}\right|_{r_{E}+\bar{h}}(h-\bar{h})^{k}\right\}+\left.\frac{\partial \zeta}{\partial \gamma} \frac{\partial \gamma}{\partial h}\right|_{r_{E}}(h-\bar{h})
$$

where the $C_{1 \mathrm{~B}}$-term is now computed as a function of the reduced heights $(h-\bar{h})$. It should be noted that Eqs. (4), (9), (13) and (14) implicitly approximate $r_{P} \approx r_{E}+h$. Numerical tests will show that this approximation is acceptable in practice.

\subsection{Numerical tests}

\subsubsection{Test design}

Equations (14) and (15) were numerically tested using EGM2008 (Pavlis et al. 2008) to $M=$ 2190 and for Taylor orders $K=0$ to 3 . For the SHS of EGM2008 functionals $\zeta$ and $\zeta_{r(k)}$, we use the state-of-the-art harmonic_synth software (Holmes and Pavlis 2008). This software makes use of accelerated routines of Holmes and Featherstone (2002a, b), allowing highlyefficient SHS at multiple computation points given in terms of regularly-spaced grids. Harmonic_synth is used here in two different modes:

- The numerically highly-efficient grid mode ("gridded computations”) to compute grids of quasigeoid heights $\zeta$ and their radial derivatives $\zeta_{r(k)}$ and

- the time-consuming "scattered-point" option to directly generate true values $\zeta^{*}$ at the 3D-locations of the topography.

Testing Eqs. (14) and (15) necessitated extension of harmonic_synth’s capability to higherorder radial derivatives $\zeta_{r(k)}$. Using the generalized expression (13), implementation is straightforward. The harmonic_synth code was also modified to allow accelerated synthesis at an arbitrarily constant height $\bar{h}$ above the ellipsoid. 
Test areas are parts of the European Alps $\left(45^{\circ}<\phi<47^{\circ}, 6^{\circ}<\lambda<9^{\circ}\right)$ with $h$ varying between $\sim 0$ and $\sim 4 \mathrm{~km}$ and parts of the Himalayas $\left(26^{\circ}<\phi<29^{\circ}, 86^{\circ}<\lambda<88^{\circ}\right)$ with $h$ varying between $\sim 0$ and $\sim 8.8 \mathrm{~km}$. The first includes the Swiss Central Alps and the latter the Mount Everest region and the flats of Nepal and North-East India, to test the methods over Earth's region with the largest variation in elevation.

Following Claessens et al. (2009), ellipsoidal heights $h$ of the topography were constructed at $120 \times 180$ regularly-spaced $1^{\prime}$ grid nodes as the sum of (i) SRTM (Shuttle Radar Topography Mission) data by Jarvis et al. (2008), and (ii) EGM2008 quasigeoid heights $\zeta_{0}$. Since SRTM elevations $H$ are mean-sea-level heights in good approximation, $H+\zeta_{0}$ yields approximate ellipsoidal heights $h$ of the topography. According to Jarvis et al. (2008), SRTM elevations are referred to EGM96 (Lemoine et al. 1998) and not EGM2008. The differences between EGM96 (to degree 360) and EGM2008 (to degree 2190) geoid undulations is estimated to be at the metre-level or below. The results of spherical harmonic synthesis are insensitive to such small changes in height, which is why this effect is considered negligible in practise.

To benchmark the approximation errors of $\zeta_{P}\left(\varphi, \lambda, r_{P}\right)$ [from Eqs. (14) and (15)] for orders $K$ $=0$ to 3 , we computed $\zeta_{P}^{*}\left(\varphi, \lambda, r_{P}\right)$ directly at the $3 \mathrm{D}$ locations $\left(\varphi, \lambda, r_{P}\right)$ with harmonic_synth's “scattered-point” option that performs full SHS point-by-point. Because of the limited number of points [21,600 $\left(\varphi, \lambda, r_{P}\right)$ triplets per test area], this is feasible within acceptable computation times (see Section 3.3.2 for run times), whilst computationally too prohibitive for millions of points. The $\zeta_{P}^{*}\left(\varphi, \lambda, r_{P}\right)$ serve as "truth" in assessing the $\zeta_{P}\left(\varphi, \lambda, r_{P}\right)$ from Eqs. (14) and (15).

\subsubsection{Test results}

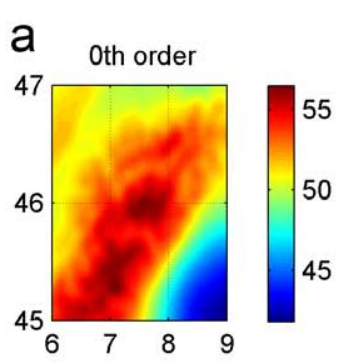

e

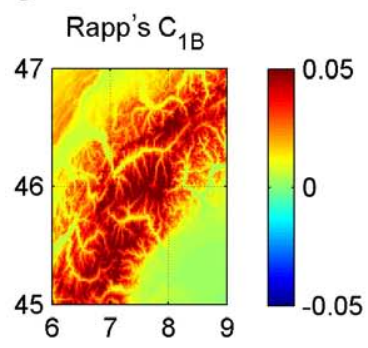

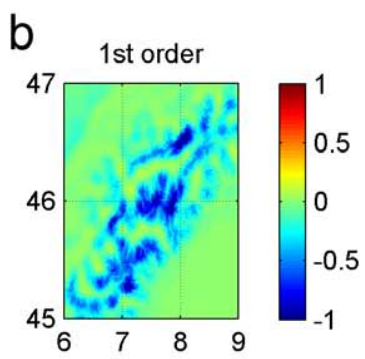

f

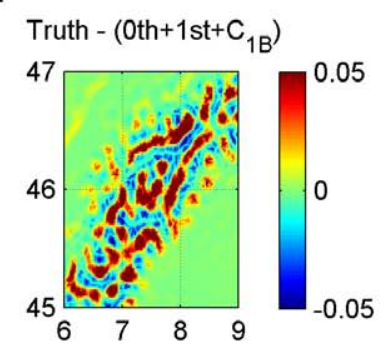

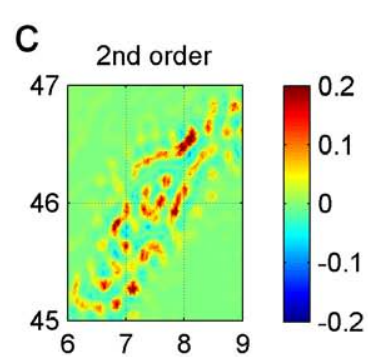

g

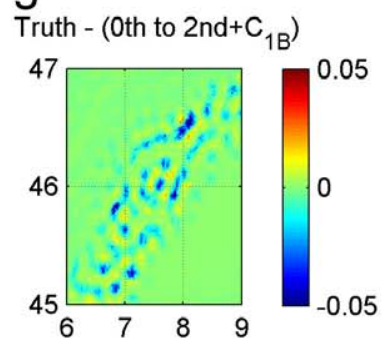

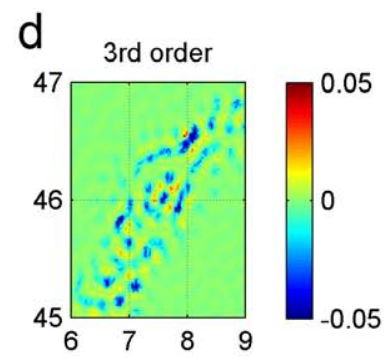

h

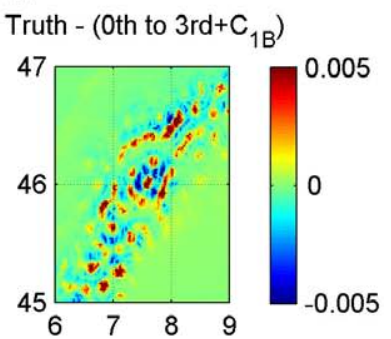


Fig.1 a Height anomalies $\zeta$ evaluated at $r_{E}$, b to $\mathbf{d} 1^{\text {st }}-, 2^{\text {nd }}$ - and $3^{\text {rd }}$-order contributions, e Rapp’s $C_{1 \mathrm{~B}}$-term, $\mathbf{f}$ to $\mathbf{h}$ Truth - (Taylor series expanded to $1^{\text {st }}, 2^{\text {nd }}$ and $3^{\text {rd }}$-order $+C_{1 \mathrm{~B}}$ ). Test area is the European Alps, no reference height used ( $\bar{h}=0 \mathrm{~m}), M=2190$, Units in metres

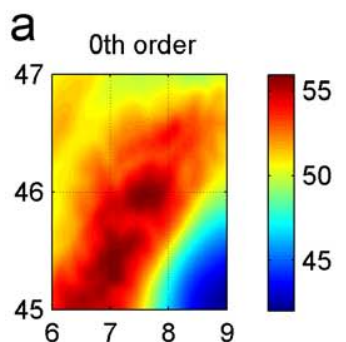

e

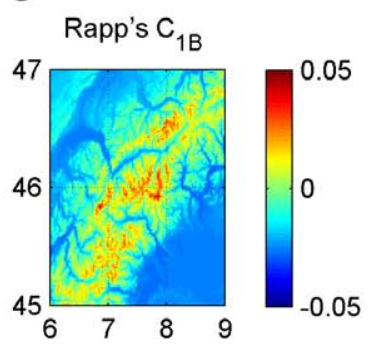

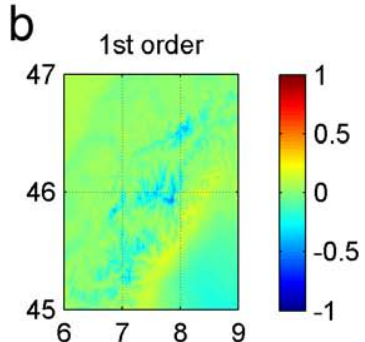

f

Truth - $\left(0\right.$ th $\left.+1 s t+C_{1 B}\right)$

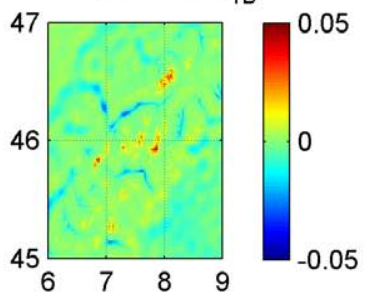

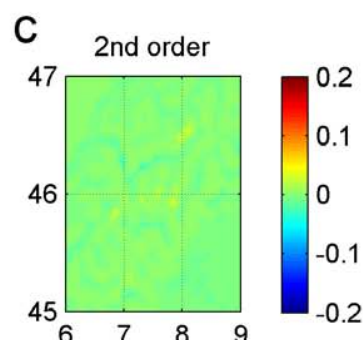
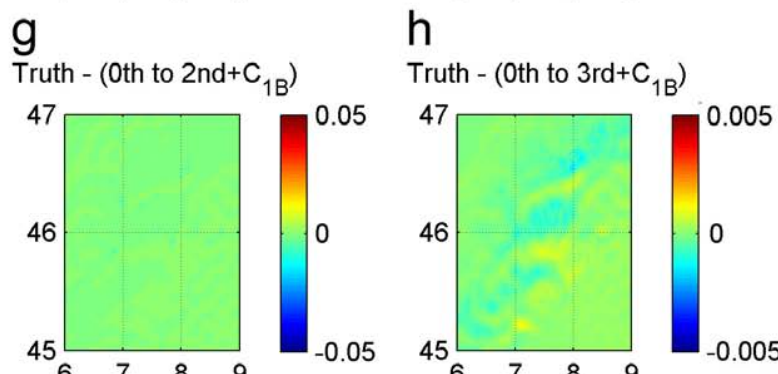

Fig.2 a Height anomalies $\zeta$ evaluated at $r_{E}+2000 \mathrm{~m}, \mathbf{b}$ to $\mathbf{d} 1^{\text {st }}-, 2^{\text {nd }}-$ and $3^{\text {rd }}$-order contributions, e Rapp's $C_{1 \mathrm{~B}}$-term, $\mathbf{f}$ to $\mathbf{h}$ Truth - (Taylor series expanded to $1^{\text {st }}, 2^{\text {nd }}$ and $3^{\text {rd }}$-order $+C_{1 \mathrm{~B}}$ ). Test area is the European Alps, $\bar{h}$ is $2000 \mathrm{~m}$ for all panels, $M=2190$, Units in metres. To allow cross-comparison the scale bars of corresponding panels in Figs. 1 and 2 are the same.

For the European area, Figs. 1a-d show the zero-th to third-order $\zeta$-contributions from Eq. (14), Fig.1e displays Rapp's $C_{1 \mathrm{~B}}$-term and Figs. 1f-1h shows the differences between the true $\zeta_{P}^{*}\left(\varphi, \lambda, r_{P}\right)$ and approximated $\zeta_{P}\left(\varphi, \lambda, r_{P}\right)$ from Eq. (14) truncated after the first-, secondand third-order. The descriptive statistics of the $\zeta_{P}^{*}\left(\varphi, \lambda, r_{P}\right)$ minus $\zeta_{P}\left(\varphi, \lambda, r_{P}\right)$ as well as the statistics of $C_{1 \mathrm{~B}}$ are reported in Table 1. From Fig. 1f, maximum errors of the first-order approach are at the dm-level over the European Alps and RMS (root-mean-square) approximation errors are $2.6 \mathrm{~cm}$ (Table 1). Not only are these approximation errors comparable to the RMS signal strength of the $C_{1 \mathrm{~B}}$-correction $(2.7 \mathrm{~cm}$, Fig. 1e), but are also at the level of EGM2008 commission errors observed for quasigeoid height differences over well-surveyed areas (which can be as low as $\sim 2-3 \mathrm{~cm}$, see Section 4 and Hirt 2011). When the first-order approach is applied over the Himalayas (Table 2), the discrepancies are as large as $\sim 1.9 \mathrm{~m}$ with an average RMS error of $\sim 16 \mathrm{~cm}$ ! These approximation errors will usually not be acceptable for accurate applications of EGM2008 and other high-degree models. 
Table 1 Descriptive statistics of the quasigeoid $\zeta$ differences for Taylor-orders 0-3 and statistics of the $C_{1 \mathrm{~B}}$ term over the European Alps

\begin{tabular}{lcccccccc}
\hline \multirow{2}{*}{ Variant } & \multicolumn{3}{c}{ No reference height } & \multicolumn{4}{c}{ Reference height $=2000$ m } \\
& min & max & mean & rms & min & max & mean & rms \\
\hline Truth - (0th order $+C_{1 \mathrm{~B}}$ ) & -1.10 & 0.11 & -0.13 & 0.223 & -0.55 & 0.22 & -0.02 & 0.086 \\
Truth - (0th+1st order $+C_{1 \mathrm{~B}}$ ) & -0.08 & 0.26 & 0.01 & 0.026 & -0.03 & 0.05 & 0.00 & 0.005 \\
Truth - (0th to 2nd order $+C_{1 \mathrm{~B}}$ ) & -0.08 & 0.03 & 0.00 & 0.006 & -0.01 & 0.00 & 0.00 & 0.001 \\
Truth - (0th to 3rd order + $C_{1 \mathrm{~B}}$ ) & -0.02 & 0.02 & 0.00 & 0.001 & 0.00 & 0.00 & 0.00 & 0.000 \\
Rapp's $C_{1 \mathrm{~B}}$ correction term & 0.00 & 0.08 & 0.02 & 0.027 & -0.03 & 0.04 & -0.01 & 0.017 \\
\hline
\end{tabular}

Statistics based on 21,600 pts, harmonic model used is EGM2008 to $M=2190$, test area is $45^{\circ}<\phi<47^{\circ}, 6^{\circ}<\lambda<9^{\circ}$ Units in metres

Table 2 Descriptive statistics of the quasigeoid $\zeta$ differences for Taylor-orders 0-3 and statistics of the $C_{1 \mathrm{~B}}$ term over the Himalayas

\begin{tabular}{lcccccccc}
\hline \multirow{2}{*}{ Variant } & \multicolumn{3}{c}{ No reference height } & \multicolumn{4}{c}{ Reference height $=\mathbf{4 0 0 0} \mathbf{~ m}$} \\
& min & max & mean & rms & min & max & mean & rms \\
\hline Truth - (0th order $+C_{1 \mathrm{~B}}$ ) & -3.95 & 0.55 & -0.34 & 0.672 & -1.76 & 0.26 & -0.29 & 0.403 \\
Truth - (0th+1st order+ $C_{1 \mathrm{~B}}$ ) & -0.47 & 1.87 & 0.03 & 0.156 & -0.12 & 0.28 & -0.01 & 0.020 \\
Truth - (0th to 2nd order $+C_{1 \mathrm{~B}}$ ) & -1.34 & 0.36 & -0.01 & 0.072 & -0.08 & 0.01 & 0.00 & 0.003 \\
Truth - (0th to 3rd order $+C_{1 \mathrm{~B}}$ ) & -0.20 & 0.84 & 0.00 & 0.031 & -0.01 & 0.02 & 0.00 & 0.001 \\
Rapp's $C_{1 \mathrm{~B}}$ correction term & -0.06 & 0.00 & -0.02 & 0.030 & -0.04 & 0.08 & 0.03 & 0.045 \\
\hline
\end{tabular}

Statistics based on 21,600 pts, harmonic model used is EGM2008 to $M=2190$, test area is $26^{\circ}<\phi<29^{\circ}, 86^{\circ}<\lambda<88^{\circ}$ Units in metres

Through inclusion of the gradients to third-order, RMS approximation errors are reduced below the cm-level (Europe, Fig. 1h) and to about $3 \mathrm{~cm}$ (Himalayas), see also Tables 1 and 2. The additional use of a constant average reference height $\bar{h}$ (2 km for the European Alps area, and $4 \mathrm{~km}$ for the Himalaya area) in Eq. (15) shortens the vertical distances $(h-\bar{h})$ over which $\zeta$ is continued to the topography. As a result, approximation errors are diminished to insignificance (0 mm RMS) over the European Alps (compare Figs.1h and 2h, see also Table 1), and to $1 \mathrm{~mm}$ RMS over the Himalaya Mountains (Table 2).

We have repeated the above tests over the European Alps using EGM2008 to degree 360 (and not to 2190 as before). The $\zeta_{P}^{*}\left(\varphi, \lambda, r_{P}\right)$ minus $\zeta_{P}\left(\varphi, \lambda, r_{P}\right)$-differences are at the level of $\sim 4$ mm RMS when using Rapp’s (1997) first-order expansion and no reference height $\bar{h}$ (this replicates the original Rapp approach). It is therefore fair to say that Rapp's first-order expansion (ibid, p. 283) was and is suitable for degree-360 $\zeta$ - and $N$-computations, whilst the second- or third-order gradient approach with reference heights $\bar{h}$ [Eq. (15)] is to be preferred for degree-2190 expansions. This is simply because with high-degree models the 
effect of gravity attenuation with height [via $\left(a / r_{P}\right)^{n}$ ] cannot be accurately 'modelled' over mountainous areas by means of linear gradients.

In the context of simulated gravity modelling, Holmes (2003, p. 31f) applied a similar strategy to use average elevations $\bar{h}$ between ellipsoid and geoid as a simple yet highlyefficient means to "increase the accuracy of the computed result". It should be noted that our tests implicitly demonstrate the approximation $r_{P} \approx r_{E}+h$ has no notable impact on our results (Tables 1, 2). As an aside, our numerical tests (Tables 1, 2) confirm the necessity to include the $C_{1 \mathrm{~B}}$-term [Eq. (6)] in Eqs. (14) and (15).

\section{The gradient approach for other functionals}

In analogy to Section 2, higher-order gradients can be used to efficiently compute other functionals of the disturbing potential at the topography. Next we present the gradient approach for gravity disturbances $\delta g$ and vertical deflections $\xi, \eta$. For the SHS expansions see, e.g., Wenzel (1985 p30f), Torge (2001), Holmes (2003, p16). Fantino and Casotto (2009) published the respective radial derivatives up to second-order, which we generalize here to arbitrary order $k$.

\subsection{Gravity disturbances $\delta g$}

Gravity disturbances $\delta g$ as the first radial derivative of the disturbing potential $T$ are obtained in spherical approximation from

$$
\begin{array}{r}
\delta g(\varphi, \lambda, r)=-\frac{\partial T}{\partial r}=\frac{G M}{r^{2}} \sum_{m=0}^{M}\left[\begin{array}{r}
\cos m \lambda \sum_{n=\mu}^{M}(n+1)\left(\frac{a}{r}\right)^{n} \bar{C}_{n m} \bar{P}_{n m}(\sin \varphi)+ \\
\sin m \lambda \sum_{n=\mu}^{M}(n+1)\left(\frac{a}{r}\right)^{n} \bar{S}_{n m} \bar{P}_{n m}(\sin \varphi)
\end{array}\right]
\end{array}
$$

where $\mu=\max (2, m)$. Eq. (16) can be evaluated with the accelerated SH routines along geodetic parallels. Expanding $\delta g$ into a Taylor series and introducing some constant ellipsoidal reference height $\bar{h}$ yields approximate $\delta g_{P}$-values at the topography

$$
\delta g_{P}\left(\varphi, \lambda, r_{P}\right) \approx \delta g_{0}\left(\varphi, \lambda, r_{E}+\bar{h}\right)+\left.\sum_{k=1}^{K} \frac{1}{k !} \frac{\partial^{k} \delta g}{\partial r^{k}}\right|_{r_{E}+\bar{h}}(h-\bar{h})^{k}
$$

where $r_{E}+\bar{h}$ is a constant quantity along geodetic parallels. The $k$-th radial derivatives $\delta g_{r(k)}=\partial^{k} \delta g / \partial r^{k}$ are obtained from the compact expression 


$$
\begin{array}{r}
\delta g_{r(k)}=\frac{\partial^{k} \delta g}{\partial r^{k}}=(-1)^{k} \frac{G M}{r^{k+2}} \sum_{m=0}^{M}\left[\cos m \lambda \sum_{n=\mu}^{M}(n+1)\left\{\prod_{i=1}^{k}(n+i+1)\right\}\left(\frac{a}{r}\right)^{n} \bar{C}_{n m} \bar{P}_{n m}(\sin \varphi)+\right. \\
\left.\quad \sin m \lambda \sum_{n=\mu}^{M}(n+1)\left\{\prod_{i=1}^{k}(n+i+1)\right\}\left(\frac{a}{r}\right)^{n} \bar{S}_{n m} \bar{P}_{n m}(\sin \varphi)\right]
\end{array}
$$

\subsection{Vertical deflections $\xi, \eta$}

The North-South vertical deflection $\xi$ is computed as a function of the latitudinal derivative $\partial T / \partial \varphi$. In spherical approximation and Molodensky definition, $\xi$ is obtained from

$$
\begin{aligned}
& \xi(\varphi, \lambda, r)=-\frac{1}{\gamma r} \frac{\partial T}{\partial \varphi}=-\frac{G M}{r^{2} \gamma} \sum_{m=0}^{M}\left[\cos m \lambda \sum_{n=\mu}^{M}\left(\frac{a}{r}\right)^{n} \bar{C}_{n m} \bar{P}_{n m}^{\prime}(\sin \varphi)+\right. \\
& \left.\sin m \lambda \sum_{n=\mu}^{M}\left(\frac{a}{r}\right)^{n} \bar{S}_{n m} \bar{P}_{n m}^{\prime}(\sin \varphi)\right]
\end{aligned}
$$

For the evaluation of first-order derivatives $\bar{P}_{n m}^{\prime}(\sin \varphi)$ of the fully-normalized Associated Legendre Function, see e.g., Bosch (2000), Holmes and Featherstone (2002b). The East-West vertical deflection $\eta$ depends on the longitudinal derivative $\partial T / \partial \lambda$ and is given through

$$
\begin{aligned}
& \eta(\varphi, \lambda, r)=-\frac{1}{\gamma r \cos \varphi} \frac{\partial T}{\partial \lambda}=-\frac{G M}{r^{2} \gamma \cos \varphi} \sum_{m=0}^{M} m\left[\cos m \lambda \sum_{n=\mu}^{M}\left(\frac{a}{r}\right)^{n} \bar{S}_{n m} \bar{P}_{n m}(\sin \varphi)-\right. \\
& \left.\sin m \lambda \sum_{n=\mu}^{M}\left(\frac{a}{r}\right)^{n} \bar{C}_{n m} \bar{P}_{n m}(\sin \varphi)\right]
\end{aligned}
$$

Series expansions are used to approximate the North-South vertical deflection

$$
\xi_{P}\left(\varphi, \lambda, r_{P}\right) \approx \xi\left(\varphi, \lambda, r_{E}+\bar{h}\right)+\left.\sum_{k=1}^{K} \frac{1}{k !} \frac{\partial^{k} \xi}{\partial r^{k}}\right|_{r_{E}+\bar{h}}(h-\bar{h})^{k}
$$

and East-West vertical deflection

$$
\eta_{P}\left(\varphi, \lambda, r_{P}\right) \approx \eta\left(\varphi, \lambda, r_{E}+\bar{h}\right)+\left.\sum_{k=1}^{K} \frac{1}{k !} \frac{\partial^{k} \eta}{\partial r^{k}}\right|_{r_{E}+\bar{h}}(h-\bar{h})^{k}
$$

at the Earth's surface, where the $k$-th radial derivative of the North-South component $\xi_{r(k)}=\partial^{k} \xi / \partial r^{k}$ and of the East-West component $\eta_{r(k)}=\partial^{k} \eta / \partial r^{k}$ are computed from the generalized expressions 


$$
\begin{aligned}
& \xi_{r(k)}=\frac{\partial^{k} \xi}{\partial r^{k}}=(-1)^{k+1} \frac{G M}{r^{k+2} \gamma} \sum_{m=0}^{M}\left[\cos m \lambda \sum_{n=\mu}^{M}\left\{\prod_{i=1}^{k}(n+i+1)\right\}\left(\frac{a}{r}\right)^{n} \bar{C}_{n m} \bar{P}_{n m}^{\prime}(\sin \varphi)+\right. \\
& \left.\sin m \lambda \sum_{n=\mu}^{M}\left\{\prod_{i=1}^{k}(n+i+1)\right\}\left(\frac{a}{r}\right)^{n} \bar{S}_{n m} \bar{P}_{n m}^{\prime}(\sin \varphi)\right] \\
& \eta_{r(k)}=\frac{\partial^{k} \eta}{\partial r^{k}}=(-1)^{k+1} \frac{G M}{r^{k+2} \gamma \cos \varphi} \sum_{m=0}^{M} m\left[\cos m \lambda \sum_{n=\mu}^{N}\left\{\prod_{i=1}^{k}(n+i+1)\right\}\left(\frac{a}{r}\right)^{n} \bar{S}_{n m} \bar{P}_{n m}(\sin \varphi)-\right. \\
& \left.\sin m \lambda \sum_{n=\mu}^{N}\left\{\prod_{i=1}^{k}(n+i+1)\right\}\left(\frac{a}{r}\right)^{n} \bar{C}_{n m} \bar{P}_{n m}(\sin \varphi)\right]
\end{aligned}
$$

\subsection{Numerical tests}

\subsubsection{Test design}

The gradient approach was tested to maximum order $K=3$ for gravity disturbances and vertical deflections over the European Alps and Himalayas test areas. The tests were performed in full analogy to the quasigeoid tests described in Section 2.4.1. Gravity disturbances [Eq. (16)] and vertical deflections [Eqs. (19), (20)] are readily computable with harmonic_synth, whereas most of their higher-order radial derivatives [Eqs. (18), (23), (24)] were not yet implemented in this software, so had to be added to the code.

Based on EGM2008 and $M=2190$, true values $\delta g_{P}^{*}\left(\varphi, \lambda, r_{P}\right), \xi_{P}^{*}\left(\varphi, \lambda, r_{P}\right)$ and $\eta_{P}^{*}\left(\varphi, \lambda, r_{P}\right)$ were generated point-by-point at the topography using harmonic_synth's "scattered-point option" [Eqs. (16), (19), (20)] and compared with $\delta g_{P}\left(\varphi, \lambda, r_{P}\right), \xi_{P}\left(\varphi, \lambda, r_{P}\right)$ and $\eta_{P}\left(\varphi, \lambda, r_{P}\right)$ as approximated with Eqs. (17), (21) and (22). All SH functionals and radial derivatives could be obtained using harmonic_synth's efficient grid mode.

\subsubsection{Test results}

The comparisons between the true and approximate functionals were drawn as a function of different Taylor-orders ( $K=0$ to 3 ), and for the variants (i) no reference height used (i.e., $\bar{h}=$ $0 \mathrm{~km}$ ), and (ii) $\bar{h}=2 \mathrm{~km}$ (Europe) and $\bar{h}=4 \mathrm{~km}$ (Himalayas). Table 3 reports the descriptive statistics of the differences true value minus approximation for the European and Table 4 for the Himalaya region. Evaluation of EGM2008 at the surface of the ellipsoid ( $\bar{h}=0$ ), and without gradients (i.e, 0th-order) gives rise to $\sim 18 \mathrm{mGal}$ RMS approximation errors for gravity disturbances and $\sim 3$ " RMS for vertical deflections over the European Alps. RMS values are as large as $\sim 50 \mathrm{mGal}$ (maximum errors of $\sim 350 \mathrm{mGal}$ ) and $\sim 7 "$ (maximum errors of $\sim 50^{\prime \prime}$ ) over the Himalayas (Table 4). What all these values reflect is the effect of gravity attenuation with height, via the spectral attenuation factor $\left(a / r_{P}\right)^{n}$ that occurs in any of the SH expansions. 
Tables 3 and 4 show that first- to third-order gradients gradually decrease the approximation errors. Using $\bar{h}=2 \mathrm{~km}$ and a third-order Taylor series reduces the approximation errors to $\sim 0.04 \mathrm{mGal}$ and $\sim 0.01 "$ over the European Alps. For the Himalaya area and $\bar{h}=4 \mathrm{~km}$, RMSerrors are found to be well below the mGal-level for gravity and smaller than $0.05^{\prime \prime}$ for deflections, which is likely a worst-case performance demonstration for the third-order gradient approach.

EGM2008 commission errors (area-weighted RMS) are globally at the level of $\sim 7 \mathrm{mGal}$ (for gravity) and 1" (for vertical deflections), but these values can vary regionally (EGM-Team 2008; Pavlis et al. 2008). Relative to the level of commission errors, approximation errors originating from the gradient approach to third-order can be safely neglected. In practical applications, limitation to second-order might be acceptable when using some average reference height $\bar{h}$, but extension to third-order reduces the approximation errors to insignificance. Hence, third-order Taylor series yield results comparable to those from rigorous point-by-point synthesis. This makes the investigated technique suitable for accurate SHS of degree-2190 vertical deflections and gravity disturbances at the Earth's surface.

The computational savings of Taylor series expansions over point-by-point synthesis are significant. Using a Sun Ultra 45 workstation (1.6 GHz with 2 GB RAM and 8 GB swap), it took a total of $\sim 5$ min to compute four grids (functional of interest and the first- to third-order derivatives) with harmonic_synth's accelerated routines, versus $\sim 150 \mathrm{~min}$ for the point-bypoint synthesis at the 3D-locations of 21,600 points. Hence the gain in efficiency is a factor $\sim 30$. Recalling that the grids were composed of merely $120 \times 180$ points, the computational savings will be much larger for regularly-spaced $(\phi, \lambda)$ or $(\varphi, \lambda)$-grids consisting of, say, thousands of points in latitude, and importantly, in longitude. The numerical efficiency of Taylor approximations over point-by-point synthesis was pointed out by Holmes (2003 p. 129ff), and our results are a confirmation of his findings.

Table 3 Descriptive statistics of the $\delta g$ (gravity disturbance) $\xi$ (NS vertical deflection) and $\eta$ (EW vertical deflection) differences for Taylor-orders 0-3 over the European Alps

\begin{tabular}{|c|c|c|c|c|c|c|c|c|c|}
\hline \multirow{2}{*}{ Functional } & \multirow{2}{*}{ Variant } & \multicolumn{4}{|c|}{ No reference height } & \multicolumn{4}{|c|}{ Reference height $=2000 \mathrm{~m}$} \\
\hline & & $\min$ & $\max$ & mean & rms & $\min$ & $\max$ & mean & rms \\
\hline$\delta g$ & Truth - 0th order & -120.4 & 53.0 & -3.9 & 18.31 & -45.3 & 25.6 & -3.2 & 7.13 \\
\hline$\delta g$ & Truth - (0th+1st order) & -21.9 & 55.2 & 1.1 & 5.92 & -7.4 & 8.8 & -0.1 & 0.95 \\
\hline$\delta g$ & Truth - (0th to 2nd order) & -18.3 & 12.3 & -0.3 & 1.61 & -1.9 & 0.6 & 0.0 & 0.12 \\
\hline$\delta g$ & Truth - (0th to 3rd order) & -4.6 & 6.0 & 0.1 & 0.37 & -0.3 & 0.3 & 0.0 & 0.04 \\
\hline$\xi$ & Truth - 0th order & -14.6 & 17.4 & -0.1 & 2.88 & -5.2 & 5.3 & -0.1 & 0.93 \\
\hline$\xi$ & Truth - (0th+1st order) & -7.4 & 7.4 & 0.0 & 0.92 & -1.0 & 1.3 & 0.0 & 0.12 \\
\hline$\xi$ & Truth - (0th to 2nd order) & -3.0 & 2.3 & 0.0 & 0.24 & -0.3 & 0.2 & 0.0 & 0.02 \\
\hline$\xi$ & Truth - (0th to 3rd order) & -0.9 & 1.0 & 0.0 & 0.06 & 0.0 & 0.1 & 0.0 & 0.01 \\
\hline$\eta$ & Truth - 0th order & -12.1 & 15.1 & 0.1 & 2.45 & -4.6 & 5.1 & 0.1 & 0.86 \\
\hline$\eta$ & Truth - (0th+1st order) & -7.8 & 5.5 & 0.0 & 0.81 & -1.2 & 0.9 & 0.0 & 0.11 \\
\hline
\end{tabular}




\begin{tabular}{llllllllll}
$\eta$ & Truth - (0th to 2nd order) & -1.9 & 2.7 & 0.0 & 0.21 & -0.2 & 0.3 & 0.0 & 0.01 \\
$\eta$ & Truth - (0th to 3rd order) & -0.9 & 0.6 & 0.0 & 0.05 & -0.1 & 0.0 & 0.0 & 0.01 \\
\hline
\end{tabular}

Statistics based on 21,600 pts, harmonic model is EGM2008 to $M=2190$, test area is $45^{\circ}<\phi<47^{\circ}, 6^{\circ}<\lambda<9^{\circ}$, Units in $\mathrm{mGal}$ for $\delta g$, units in arc seconds for $\xi, \eta$

Table 4 Descriptive statistics of the $\delta g$ (gravity disturbance), $\xi$ (NS vertical deflection) and $\eta$ (EW vertical deflection) differences for Taylor-orders 0-3 over the Himalayas

\begin{tabular}{cccccccccc}
\hline $\begin{array}{c}\text { Func- } \\
\text { tional }\end{array}$ & \multirow{2}{*}{ Variant } & \multicolumn{3}{c}{ No reference height } & \multicolumn{4}{c}{ Reference height $=\mathbf{4 0 0 0}$ m } \\
\hline$\delta g$ & Truth - 0th order & -350.8 & 165.3 & -7.1 & 49.9 & -110.6 & 45.4 & -6.7 & 14.69 \\
$\delta g$ & Truth - (0th+1st order) & -157.8 & 410.2 & 2.7 & 34.7 & -22.1 & 49.0 & -0.3 & 3.31 \\
$\delta g$ & Truth - (0th to 2nd order) & -358.9 & 110.5 & -1.4 & 19.5 & -19.6 & 4.3 & -0.1 & 0.90 \\
$\delta g$ & Truth - (0th to 3rd order) & -61.7 & 255.6 & 0.6 & 9.0 & -1.8 & 7.0 & 0.0 & 0.24 \\
& & & & & & & & & \\
$\xi$ & Truth - 0th order & -35.8 & 32.6 & 0.1 & 6.6 & -11.1 & 6.9 & -0.3 & 1.90 \\
$\xi$ & Truth - (0th+1st order) & -35.3 & 41.3 & 0.0 & 4.8 & -3.5 & 4.1 & 0.0 & 0.46 \\
$\xi$ & Truth - (0th to 2nd order) & -32.4 & 31.4 & 0.0 & 2.7 & -1.4 & 1.5 & 0.0 & 0.13 \\
$\xi$ & Truth - (0th to 3rd order) & -20.6 & 18.5 & 0.0 & 1.2 & -0.5 & 0.4 & 0.0 & 0.04 \\
& & & & & & & & & \\
$\eta$ & Truth - 0th order & -43.3 & 50.3 & 0.1 & 7.8 & -10.6 & 10.9 & -0.1 & 1.89 \\
$\eta$ & Truth - (0th+1st order) & -37.7 & 32.6 & 0.0 & 5.2 & -3.5 & 2.6 & 0.0 & 0.43 \\
$\eta$ & Truth - (0th to 2nd order) & -20.6 & 26.6 & 0.0 & 2.8 & -1.1 & 1.2 & 0.0 & 0.11 \\
$\eta$ & Truth - (0th to 3rd order) & -17.6 & 14.1 & 0.0 & 1.2 & -0.4 & 0.3 & 0.0 & 0.03 \\
\hline
\end{tabular}

Statistics based on 21,600 pts, harmonic model is EGM2008 to $M=2190$, test area is $26^{\circ}<\phi<29^{\circ}, 86^{\circ}<\lambda<88^{\circ}$, Units in $\mathrm{mGal}$ for $\delta g$, units in arc seconds for $\xi, \eta$

\section{Application example}

The gradient approach, as described and tested in Sections 2 and 3, enables accurate yet efficient SHS of high-degree GGM functionals at densely-spaced points located at Earth's surface. Because the incorporation of heights is separated from the actual SHS, the 3D point density can be arbitrarily high, e.g., up to the spatial resolution of elevation models. As a demonstration of feasibility, we have applied the gradient approach for the construction of an ultra-high resolution model of surface gravity field functionals over the European Alps, that we name here EurAlpGM2011 (European Alps Gravity Model 2011). The EurAlpGM2011 model is freely available from http://www.geodesy.curtin.edu.au/research/models.

\subsection{Construction of EurAlpGM2011}

EurAlpGM2011 is a composite model that uses EGM2008 (Pavlis et al. 2008) in spectral band 2 to 2190 and topography-implied gravity effects from residual terrain model (RTM) data beyond harmonic degree 2190 . Over a $4^{\circ} \times 12^{\circ}$ target area covering the European Alps $\left(44^{\circ}<\phi<48^{\circ}, 5^{\circ}<\lambda<17^{\circ}\right)$, ellipsoidal heights $h$ of the topography were constructed from SRTM (V4.1 250m-release by Jarvis et al. 2008) and EGM2008 quasigeoid heights (cf. Section 2.4) at 7.2" spatial resolution, corresponding to $12 \times 10^{6}$ points. 
EGM2008 functionals $\zeta^{E G M 2008}, \delta g^{E G M 2008}, \xi^{E G M 2008}$ and $\eta^{E G M 2008}$ as well as their first- to third-order radial derivatives were computed at $\bar{h}=2 \mathrm{~km}$ above the GRS80 reference ellipsoid at regularly-spaced 36" grid points, and bicubically interpolated at the $(\phi, \lambda)$ locations of the 7.2" elevation grid. The series expansions [Eqs. (15), (17), (21) and (22)] were then evaluated with the $h-\bar{h}$ values to third-order, yielding $\zeta_{P}^{E G M 2008}, \quad \delta g_{P}^{E G M 2008}$ , $\xi_{P}^{E G M} 2008$ and $\eta_{P}^{E G M 2008}$ at the $12 \times 10^{6}$ points of the topography. The North-South deflection $\xi$ was corrected for the curvature of the normal plumbline (Jekeli 1999; Hirt et al. 2010a)

$\delta \xi \approx 0.17 " \cdot h[\mathrm{~km}] \cdot \sin 2 \phi$,

an effect that reaches up to $\sim 0.8^{\prime \prime}$ (RMS of $\sim 0.2^{\prime \prime}$ ) in our target area. The $\delta \xi$ were added to Eq. (21) to convert $\xi^{E G M 2008}$ from Molodensky's to Helmert's definition (Jekeli 1999). As a simplification, the spherically approximated $\delta g$ and $\xi$ [Eqs. (16) and (19)] are not corrected here for the ellipsoidal effect, i.e., the difference between spherical and ellipsoidal approximation (e.g., Claessens 2006, p. 89; Jekeli 1999). The RMS-signal strength of the ellipsoidal effect on $\delta g$ is less than $0.1 \mathrm{mGal}$ globally (EGM2008 band 2 to 2190) and the RMS is below 0.1" for $\xi$ (Hirt et al. 2010a), whilst the $\eta$-component is unaffected (Jekeli 1999).

Over rugged terrain, EGM2008 omits significant short-wavelength (i.e., scales shorter $\sim 5^{\prime}$ ) gravity signals originating from the gravitational attraction of the topography (e.g., Hirt 2010; Hirt et al. 2010b; Hirt et al. 2011). The EGM2008 surface functionals were therefore spectrally augmented using topography-implied gravity-effects from residual terrain model data (RTM, Forsberg 1984). RTM was used in the development of EGM2008 (e.g., Pavlis et al. 2007) and tested as augmentation of EGM2008 (Hirt 2010, Hirt et al. 2010b, Gruber et al. 2011b, Hirt et al. 2011; Filmer 2011; Marti et al. 2011).

Following Hirt (2010), RTM elevations are constructed as difference of SRTM (Jarvis et al. 2008) and DTM2006.0 (Pavlis et al. 2007) spherical harmonic elevations to degree 2160, and converted to RTM functionals $\zeta^{R T M}, \delta g^{R T M}, \xi^{R T M}$ and $\eta^{R T M}$ at the same grid points used for EGM2008 synthesis. The conversion of RTM elevations to RTM functionals is based on brute-force numerical prism integration of gravity-effects using Forsberg's TC-software (Forsberg 1984), along with a constant mass-density assumption of $2670 \mathrm{~kg} \mathrm{~m}^{-3}$ and an integration radius of $200 \mathrm{~km}$ for each computation point. A justification for this approach and parameters used is found in Hirt (2010) and Hirt et al. (2010b).

EurAlpGM2011 is the sum of EGM2008 (evaluated with the gradient approach at the topography) and the RTM functionals which serve as high-frequency augmentation beyond the EGM2008 resolution, at spatial scales of $\sim 7^{\prime \prime}$ to $\sim 5^{\prime}$. EurAlpGM2011 resolves the gravity field over the European Alps at ultra-high spatial resolution of 7.2" at the surface of the SRTM-topography. The EurAlpGM2011 quasigeoid heights $\left(\zeta_{P}^{E G M}{ }^{2008}+\zeta^{R T M}\right)$ are shown in 
Fig. 3, gravity disturbances $\left(\delta g_{P}^{E G M}{ }^{2008}+\delta g^{R T M}\right)$ in Fig. 4, North-South vertical deflections $\left(\xi_{P}^{E G M 2008}+\xi^{R T M}\right)$ in Fig. 5 and East-West vertical deflections $\left(\eta_{P}^{E G M 2008}+\eta^{R T M}\right)$ in Fig. 6. Additionally, the total vertical deflections $\varepsilon=\sqrt{\left(\xi_{P}^{E G M 2008}+\xi^{R T M}\right)^{2}+\left(\eta_{P}^{E G M 2008}+\eta^{R T M}\right)^{2}}$ are displayed in Fig. 7. Specifically the maps of the gravity disturbances and vertical deflections show the high spatial variability of the entire Alpine gravity field, at a detail resolution of $\sim 220 \mathrm{~m}$ in latitude and $\sim 155 \mathrm{~m}$ in longitude.

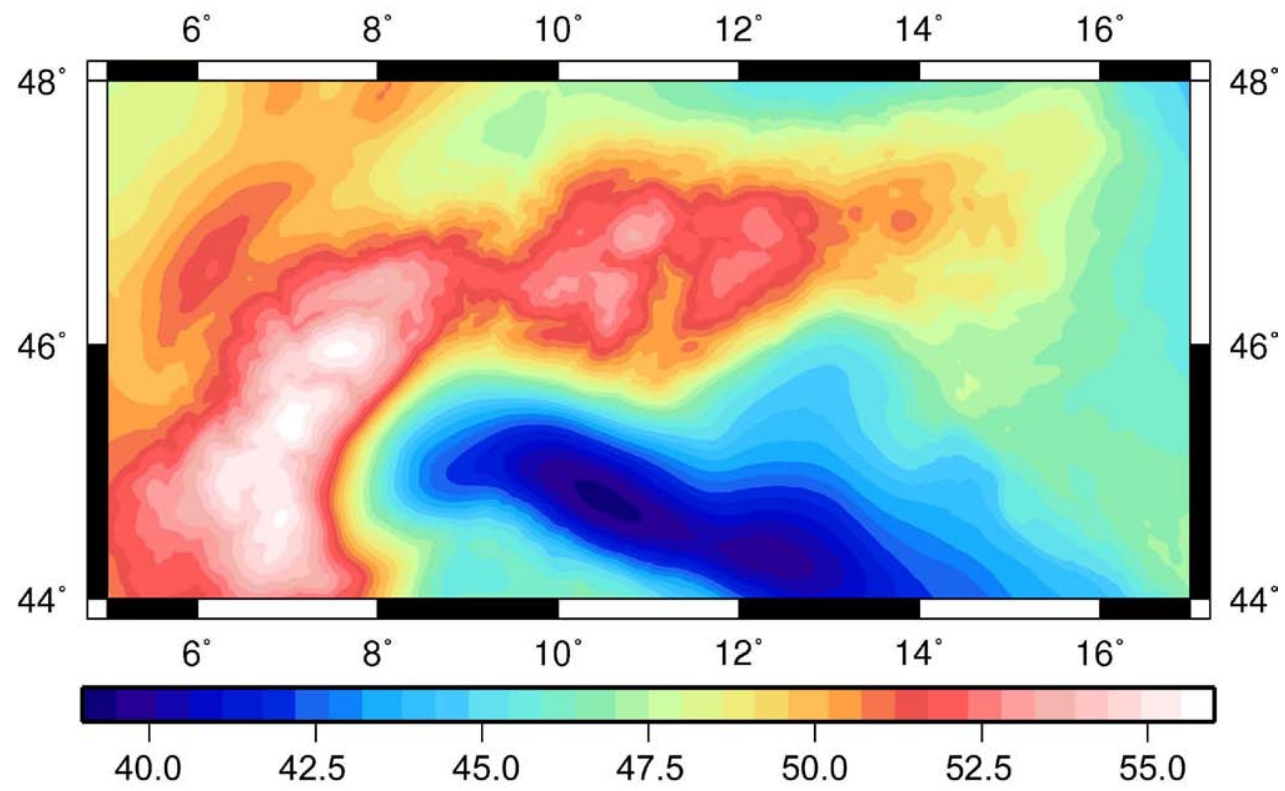

Fig. 3 EurAlpGM2011 quasigeoid undulations over the European Alps, 7.2 arc second spatial resolution, Mercator projection centred at $11^{\circ}$ longitude, unit in metres

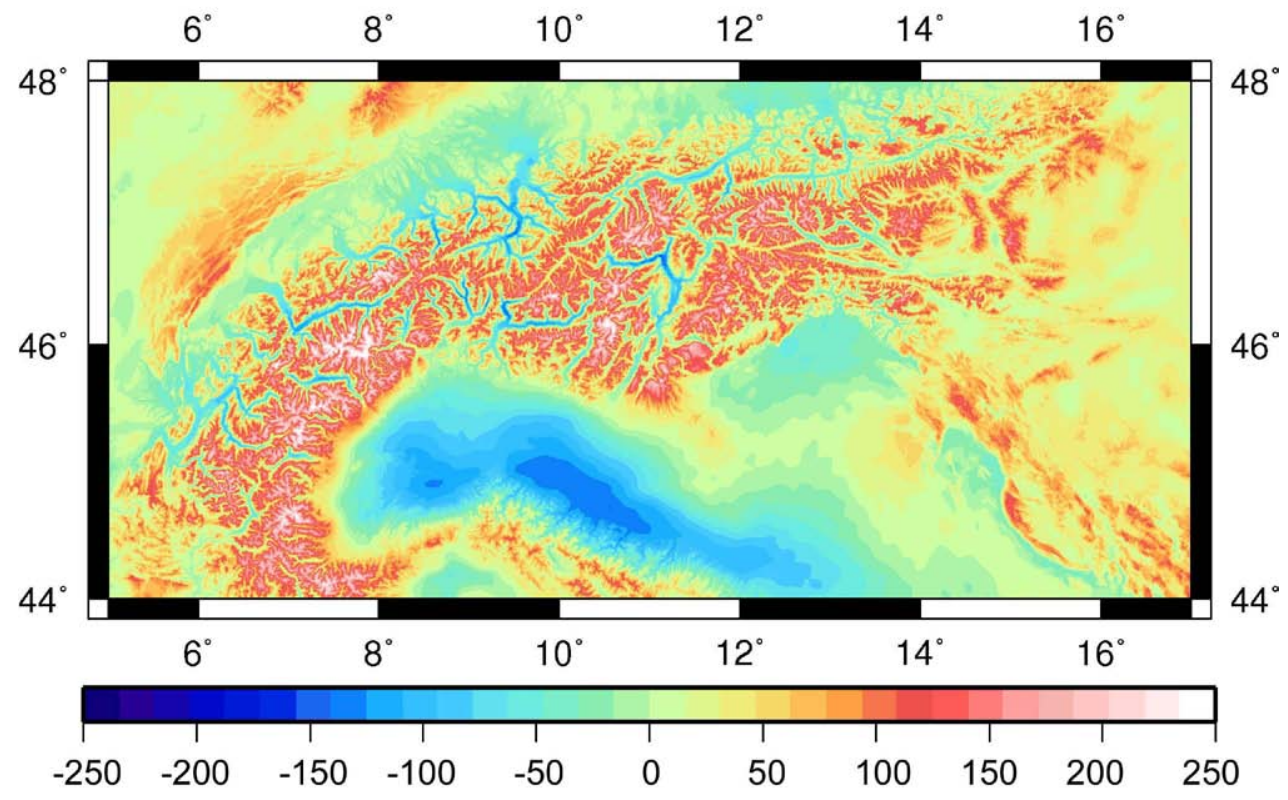

Fig. 4 EurAlpGM2011 gravity disturbances, unit in mGal 


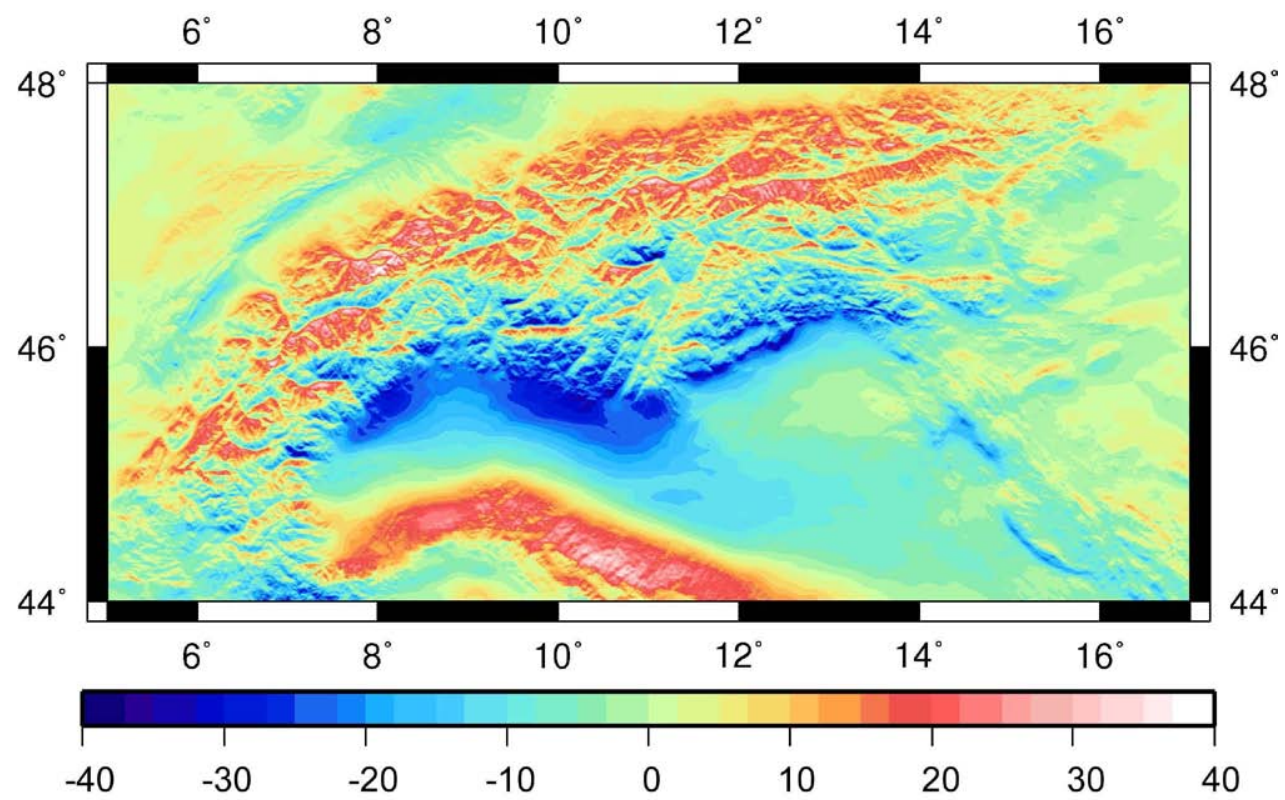

Fig. 5 EurAlpGM2011 North-South vertical deflections, unit in arc seconds

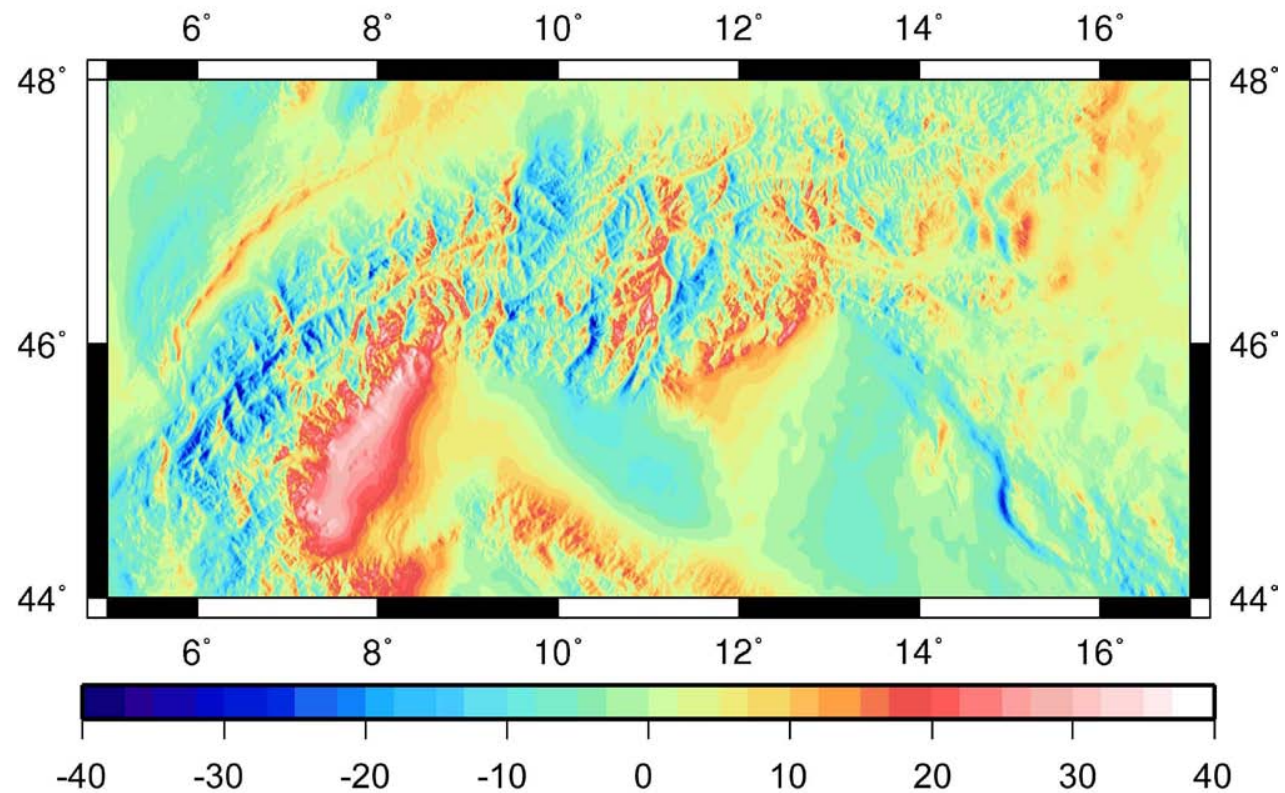

Fig. 6 EurAlpGM2011 East-West vertical deflections, unit in arc seconds 


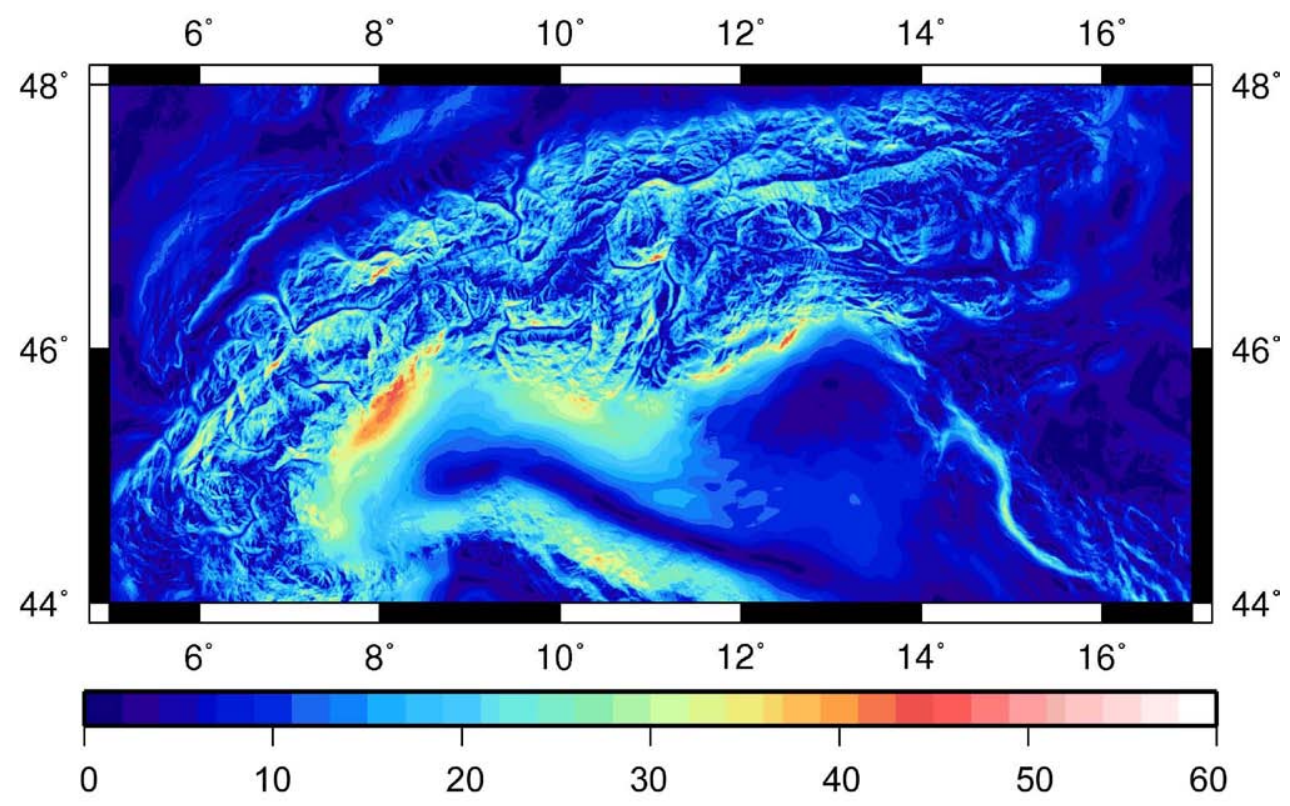

Fig. 7 Total vertical deflections over the European Alps, unit in arc seconds

\subsection{Evaluation of EurAlpGM2011}

To evaluate the EurAlpGM2011 construction, ground-control stations were available as follows:

- 34 quasigeoid heights from GPS/levelling (Ihde and Sacher 2002) over Southern Germany,

- 31598 terrestrial gravity measurements over Switzerland (Swiss Geodetic Commission, Marti 2004), and

- 690 astrogeodetically observed vertical deflections (data from Swiss Geodetic Commission, B. Bürki, author's own observations, see Hirt et al. 2010a) over Switzerland and Southern Germany.

The quasigeoid height differences from the GPS/levelling data are accurate to few $\mathrm{cm}$ (Hirt 2011, see also Table 5), the accuracy of the gravity observations is at the $0.1 \mathrm{mGal}$ level and better (U. Marti, pers. comm. 2010) and the accuracy of the vertical deflections varies between $\sim 0.1^{\prime \prime}$ and $\sim 0.5 "$ (Hirt et al. 2010a).

The EurAlpGM2011 functionals (Figs. 3 to 6) were bicubically interpolated at the $(\phi, \lambda)$ locations of the ground-control stations. A bias-fit was applied for the GPS/levelling points to remove (constant) vertical datum offsets, which is why the quasigeoid heights are tested here in a relative sense. The terrestrial gravity was converted to gravity disturbances by subtracting GRS80 normal gravity (Torge 2001, p. 106 and p. 110). The astrogeodetic deflections and GPS/levelling quasigeoid heights represent independent ground-control while inter-dependencies between EGM2008 and the Swiss gravity data exist (e.g., Hirt et al. 2011). 
The RMS of the differences "observation minus EurAlpGM2011 functional” are $2 \mathrm{~cm}$ for the quasigeoid height differences, $4.6 \mathrm{mGal}$ for the gravity disturbances and 1.3" for both vertical deflection components (Table 5). The descriptive statistics of the modelling variants "EGM2008 evaluated at the ellipsoid” [Eqs. (3), (16), (19) and (20), without height information and gradients] exhibit considerably larger RMS-errors $(3 \mathrm{~cm}, 9.4 \mathrm{mGal}$ and 2.1"). This behaviour demonstrates the necessity to incorporate height data in high-degree SHS if surface quantities are required. For the sake of completeness, the descriptive statistics is also given for the variants "with and without RTM augmentation", showing the benefits conferred by RTM-augmentation of EGM2008 over rugged terrain (cf. Hirt 2010; Hirt et al. 2010b; Hirt et al. 2011).

Table 5 Descriptive statistics of ground-control observations (quasigeoid $\zeta$, gravity disturbances $\delta g$, vertical deflections $\xi, \eta$ ) minus modelled quantities from four variants (EGM2008 evaluated at the ellipsoid/evaluated at the topography, and RTM augmentation applied/not applied)

\begin{tabular}{|c|c|c|c|c|c|c|c|}
\hline $\begin{array}{l}\text { Func- } \\
\text { tional }\end{array}$ & Difference & $\begin{array}{c}\text { EGM2008 } \\
\text { evaluation at }\end{array}$ & $\begin{array}{c}\text { RTM } \\
\text { augmentation }\end{array}$ & Min & Max & Mean & RMS \\
\hline$\zeta$ & Obs-EurAlpGM2011 & topography $^{+}$ & yes & -0.06 & 0.04 & 0.00 & 0.020 \\
\hline$\zeta$ & Obs-EGM2008/RTM & ellipsoid & yes & -0.06 & 0.06 & 0.00 & 0.030 \\
\hline$\zeta$ & Obs-EGM2008 & topography $^{+}$ & no & -0.08 & 0.14 & 0.00 & 0.041 \\
\hline$\zeta$ & Obs-EGM2008 & ellipsoid & no & -0.10 & 0.16 & 0.00 & 0.051 \\
\hline$\delta g$ & Obs- EurAlpGM2011 & topography $^{+}$ & yes & -91 & 29 & -1 & 4.6 \\
\hline$\delta g$ & Obs-EGM2008/RTM & ellipsoid & yes & -96 & 60 & 0 & 9.4 \\
\hline$\delta g$ & Obs-EGM2008 & topography $^{+}$ & no & -225 & 95 & -18 & 39.5 \\
\hline$\delta g$ & Obs-EGM2008 & ellipsoid & no & -256 & 120 & -17 & 40.3 \\
\hline$\xi$ & Obs- EurAlpGM2011 & topography $^{+}$ & yes & -5 & 5 & 0 & 1.3 \\
\hline$\xi$ & Obs-EGM2008/RTM & ellipsoid & yes & -11 & 10 & 0 & 2.1 \\
\hline$\xi$ & Obs-EGM2008 & topography $^{+}$ & no & -15 & 16 & 0 & 3.7 \\
\hline$\xi$ & Obs-EGM2008 & ellipsoid & no & -18 & 12 & 0 & 4.1 \\
\hline$\eta$ & Obs- EurAlpGM2011 & topography $^{+}$ & yes & -5 & 5 & 0 & 1.3 \\
\hline$\eta$ & Obs-EGM2008/RTM & ellipsoid & yes & -7 & 11 & 0 & 2.1 \\
\hline$\eta$ & Obs-EGM2008 & topography $^{+}$ & no & -12 & 16 & 0 & 3.6 \\
\hline$\eta$ & Obs-EGM2008 & ellipsoid & no & -13 & 21 & 1 & 4.1 \\
\hline
\end{tabular}

EurAlpGM2011 is EGM2008 (evaluated at the topography ${ }^{+}$) with RTM-augmentation. The ${ }^{+}$denotes evaluation of EGM2008 at the height of the topography using the third-order gradient approach. Units in metres ( $\zeta$ ), mGal $(\delta g)$ and arc seconds $(\xi, \eta)$

\subsection{Application of EurAlpGM2011}

An ultra-high resolution composite model of surface gravity functionals such as EurAlpGM2011 can be applied for gravity field statistics, allowing analysis of signal strengths and extreme values. From Fig. 4, gravity disturbances can be as large as $290 \mathrm{mGal}$ in our test area (near $45.93^{\circ} \mathrm{N}, 7.73^{\circ} \mathrm{E}$ ) and maximum vertical deflections are expected to be 
about or in excess of $50^{\prime \prime}$ near $46.59^{\circ} \mathrm{N}, 8.01^{\circ} \mathrm{E}$ (Fig. 7). Models like EurAlpGM2011 are suitable for planning of gravity field surveys, or detection of gross-errors in gravity data bases, and the construction principles are useful for simulated gravity field modelling. It can also be a convenient source of (i) quasigeoid height differences for GNSS (global navigation satellite system)-based height transfer (e.g., Hirt et al. 2010b), (ii) vertical deflections for reduction of survey data (e.g., Featherstone and Rüeger 2000) and (iii) medium-accuracy gravity values for the re-construction of gravity values at surveying benchmarks (Filmer 2011) and the computation of levelling reductions (Meyer et al. 2006), without the need to perform observations. Importantly, the gradient approach investigated in this paper is a key to constructing similar models over other parts of Earth which may be beneficial for a range of potential applications.

\section{Conclusions}

This study investigated the gradient approach for efficient and accurate SHS of surface gravity field quantities, offering a pragmatic solution to the high-degree SHS height problem. Taylor series were formulated to arbitrary order for quasigeoid heights, gravity disturbances and vertical deflections. Evaluation of the SHS expansions at some constant reference height above the ellipsoid allows accelerated SHS along geodetic parallels, and keeps approximation errors small. Even over the roughest regions of Earth, third-order expansions produce approximation errors far below the EGM2008 commission errors, so are sufficient for practical applications.

Using linear-gradients-only (the original Rapp approach) along with degree-2190 GGMs over mountainous areas produces cm- to dm-level RMS approximation errors for the 'upwardcontinued' quasigeoid heights. The inclusion of second- to third-order terms and use of some constant reference height is therefore recommended. Because in the gradient approach elevation data is treated isolated from the actual SHS, the density of evaluation points can be easily increased up to the spatial resolution of elevation models. This was demonstrated by applying the gradient approach for the construction of ultra-high resolution surface gravity field quantities over the regional-scale European Alps area. Ground-control comparisons corroborated the importance of evaluating high-degree GGMs at the Earth's surface if surface functionals - such as quasigeoid heights, gravity disturbances and (Helmert) vertical deflections - are required.

In principle, the gradient approach can be utilized to construct ultra-high resolution maps of gravity field quantities over all land areas of Earth with SRTM or other high-resolution elevation data available. With the techniques investigated in this study it is now also possible - within acceptable computation times - to use continental-scale ground-control gravity data sets of $\sim 10^{6}$ or more points, e.g., over Australia, USA or Canada, for rigorous comparisons with existing and future high-resolution GGMs (in the past, the related computational efforts were too prohibitive, cf. Claessens et al. 2009). 
As a general conclusion the gradient approach can be used along with any present and future high-performance SHS algorithm capable of computing higher-order radial derivatives of the gravity field quantity of interest. With the principles described in this paper, the gradient approach can be adapted to other gravity field quantities (such as from gradiometry, e.g., in the context of the GOCE satellite mission) that were not dealt with in Sections 2 and 3. For future application of the gradient approach along with extremely high-degree GGMs (beyond degree 2190), radial derivatives higher than third-order might be required which can be computed with the generalized expressions for $k$-th order radial derivatives, as summarized in Table 6.

Acknowledgements This research was supported through ARC Discovery Project Grant DP0663020. Sincere thanks go to the providers of ground-control data (Uwe Schirmer and Bundesamt für Kartographie und Geodäsie, Germany; Urs Marti and Beat Bürki, Swiss Geodetic Comission), and to the reviewers for their comments on the manuscript. I would like to gratefully thank Will Featherstone for his mentoring over the past years. Some figures were produces with the Generic Mapping Tools GMT (Wessel and Smith 1998). This is The Institute for Geoscience Research publication Nr 409.

Table 6 Radial derivatives of arbitrary order $k$ for height anomalies $\zeta$, gravity disturbances $\delta g$ and vertical deflections $\xi, \eta$

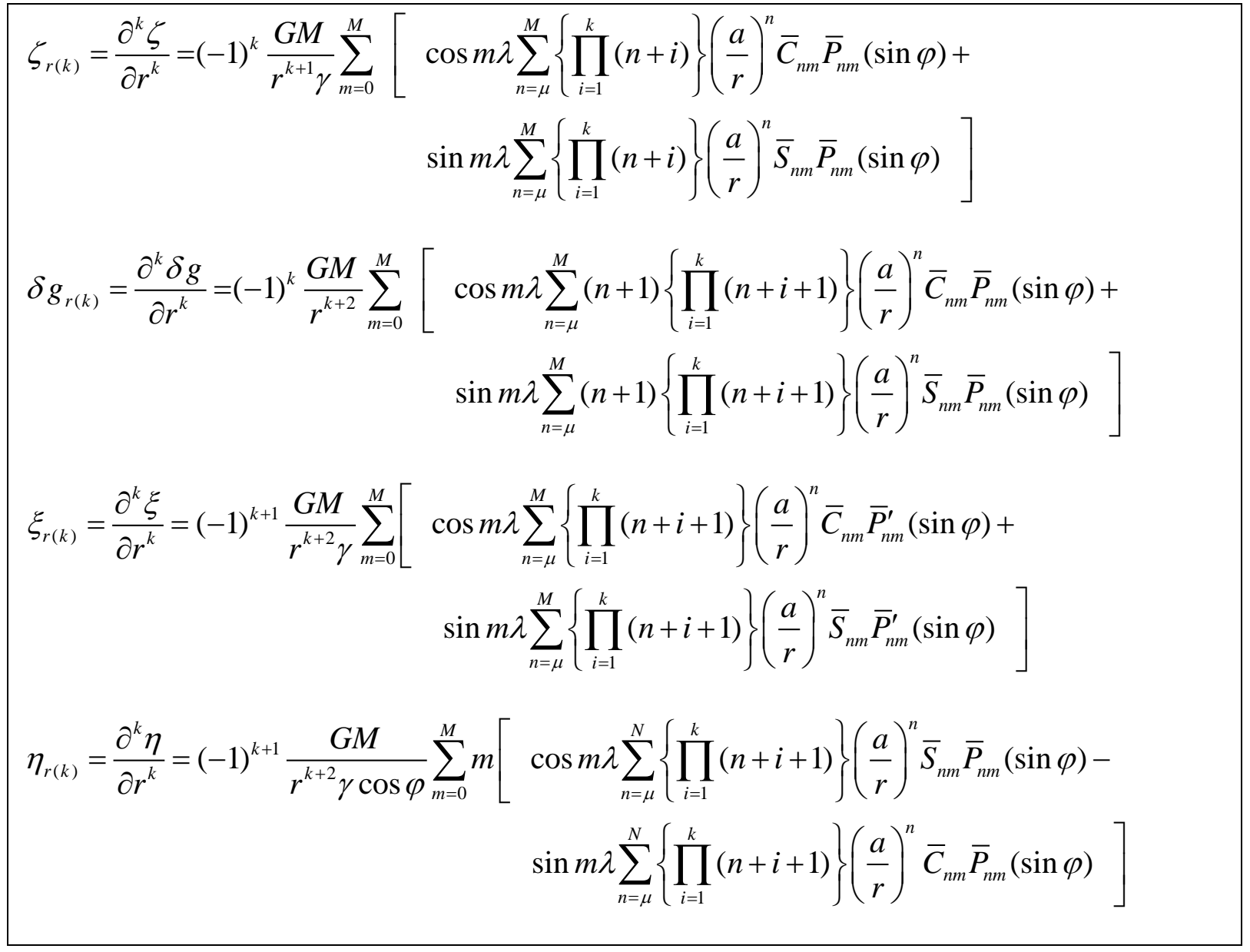

$k \geq 1, \quad \mu=\max (2, m)$ 


\section{References}

Abd-Elmotaal HA (1997) An efficient technique for the computation of the gravimetric quantities from geopotential earth models. Paper presented at IAG Scientific Assembly, 3.-9. September, Rio de Janeiro, Brazil

Ågren J (2004) The analytical continuation bias in geoid determination using potential coefficients and terrestrial gravity data. J Geod 78(4-5): 314-332. doi 10.1007/s00190004-0395-0

Bethencourt A, Wang J, Rizos C, Kearsley AHW (2005) Using personal computers in spherical harmonic synthesis of high degree Earth geopotential models. Paper presented at Dynamic Planet 2005, Cairns, Australia, 22-26 August 2005

Bosch W (2000) On the computation of derivatives of Legendre functions. Phys Chem Earth A: Solid Earth and Geodesy 25(9-11): 655-659. doi:10.1016/S1464-1895(00)00101-0

Casotto S, Fantino E (2007) Evaluation of methods for spherical harmonic synthesis of the gravitational potential and its gradients. Advances in Space Research 40(1):69-75. doi:10.1016/j.asr.2007.01.021

Claessens SJ (2006) Solutions to Ellipsoidal Boundary Value Problems for Gravity Field Modelling. PhD thesis, Department of Spatial Sciences, Curtin University of Technology, Perth, Australia, 220 pp

Claessens SJ, Featherstone WE, Anjasmara IM, Filmer MS (2009) Is Australian data really validating EGM2008 or is EGM2008 just in/validating Australian data. In: Newton's Bulletin Issue $\mathrm{n}^{\circ}$ 4, April 2009 ISSN 1810-8555, Publication the International Association of Geodesy and International Gravity Field Service, pp 207-251

EGM-Team (2008) Description of files Containing Propagated Error Estimates From EGM2008 on Global 5'x5' Grids. U.S. National Geospatial-Intelligence Agency (NGA)

Fantino E, Casotto S (2009) Methods of harmonic synthesis for global geopotential models and their first-, second- and third-order gradients. J Geod 83(7):595-619. doi 10.1007/s00190-008-0275-0

Featherstone WE, Kirby JF (1998) Estimates of the separation between the geoid and quasigeoid over Australia. Geomatics Research Australasia 68(5): 75-86

Featherstone WE, Rüeger JM (2000) The importance of using deviations of the vertical for the reduction of survey data to a geocentric datum. The Australian Surveyor 45(2):4661, Erratum in The Australian Surveyor, 47(1):7

Filmer MS (2011) An examination of the Australian Height datum. PhD thesis, Department of Spatial Sciences, Curtin University of Technology, Perth

Flury J, Rummel, R (2009) On the geoid-quasigeoid separation in mountain areas. J Geod 83(9):829-847. DOI: 10.1007/s00190-009-0302-9

Forsberg R (1984) A study of terrain reductions, density anomalies and geophysical inversion methods in gravity field modelling. Report 355, Department of Geodetic Science and Surveying, Ohio State University, Columbus

Förste C, Bruinsma S, Shako R, Marty JC, Flechtner F, Abrikosov O, Dahle C, Lemoine JM, Neumayer H, Biancale R, Barthelmes F, König R, Balmino G (2011) EIGEN-6 - A new combined global gravity field model including GOCE data from the collaboration of 
GFZ-Potsdam and GRGS-Toulouse. Geophysical Research Abstracts Vol. 13, EGU2011-3242-2, 2011 EGU General Assembly, Vienna

Fukushima T (2011) Numerical computation of spherical harmonics of arbitrary degree and order by extending exponent of floating point numbers. J Geod, online first. DOI: 10.1007/s00190-011-0519-2

Gruber C, Novák P, Sebera J (2011a) FFT-based high-performance spherical harmonic transformation. Stud Geoph Geod 55(3):489-500. Doi: 10.1007/s11200-011-0029-y

Gruber T, Visser PNAM, Ackermann C, Hosse M (2011b) Validation of GOCE gravity field models by means of orbit residuals and geoid comparisons. J Geod 85(11): 845-860. doi: 10.1007/s00190-011-0486-7

Heiskanen, WA, Moritz H (1967) Physical Geodesy, Freeman, San Francisco

Hirt C (2010) Prediction of vertical deflections from high-degree spherical harmonic synthesis and residual terrain model data. J Geod 84(3):179-190. doi 10.1007/s00190009-0354-X

Hirt C, Marti U, Bürki B, Featherstone WE (2010a) Assessment of EGM2008 in Europe using accurate astrogeodetic vertical deflections and omission error estimates from SRTM/DTM2006.0 residual terrain model data. J Geophys Res Solid Earth 115, B10404. doi:10.1029/2009JB007057

Hirt C, Featherstone WE, Marti U (2010b) Combining EGM2008 and SRTM/DTM2006.0 residual terrain model data to improve quasigeoid computations in mountainous areas devoid of gravity data. J Geod 84(9): 557-567. doi 10.1007/s00190-010-0395-1

Hirt, C, Gruber T, Featherstone WE (2011) Evaluation of the first GOCE static gravity field models using terrestrial gravity, vertical deflections and EGM2008 quasigeoid heights. J Geod 85(10): 723-740. doi: 10.1007/s00190-011-0482-y

Hirt C (2011) Assessment of EGM2008 over Germany using accurate quasigeoid heights from vertical deflections, GCG05 and GPS/levelling. Zeitschrift für Geodäsie, Geoinformation und Landmanagement (zfv) 136(3): 138-149

Holmes SA (2003) High degree spherical harmonic synthesis for simulated earth gravity modelling. PhD Thesis, Department of Spatial Sciences, Curtin University of Technology, Perth, Australia, $171 \mathrm{pp}$

Holmes SA, Featherstone WE (2002a) A unified approach to the Clenshaw summation and the recursive computation of very high degree and order normalized associated Legendre functions. J Geod 76(5):279-299. doi: 10.1007/s00190-002-0216-2

Holmes, SA, Featherstone WE (2002b) SHORT NOTE: Extending simplified high-degree synthesis methods to second latitudinal derivatives of geopotential. J Geod 76(8): 447450, doi: 10.1007/s00190-002-0268-3

Holmes SA, Featherstone WE (2002c) A simple and stable approach to high degree spherical harmonic synthesis. In: Adam, J. and Schwarz, K.P (eds) Vistas for Geodesy in the New Millennium, Springer, Berlin, 259-264

Holmes SA, Pavlis NK (2008) Spherical harmonic synthesis software harmonic_synth. http://earth-info.nga.mil/GandG/wgs84/gravitymod/new_egm/new_egm.html

Ihde J, Sacher M (2002) EUREF Publikation 11/I. Mitteilungen des Bundesamtes für Kartographie und Geodäsie, Band 25, Frankfurt/Main, ISBN 3-89888-869-X 
Jarvis A, Reuter HI, Nelson A Guevara E (2008) Hole-filled SRTM for the globe Version 4. Available from the CGIAR-SXI SRTM 90m database: http://srtm.csi.cgiar.org

Lemoine FG, Kenyon SC, Factor JK, Trimmer RG, Pavlis NK., Chinn DS, Cox CM., Klosko SM., Luthcke SB, Torrence MH, Wang YM, Williamson RG, Pavlis EC, Rapp RH, Olson TR (1998) The development of the joint NASA GSFC and the National Imagery and Mapping Agency (NIMA) geopotential model EGM96, NASA/TP-1998-206861

Jekeli C (1999) An analysis of vertical deflections derived from high-degree spherical harmonic models. J Geod 73(1):10-22, doi: 10.1007/s001900050213

Jekeli C (2006) Geometric Reference Systems in Geodesy. Report, Division of Geodesy and Geospatial Science, School of Earth Sciences, Ohio State University

Jekeli C, Lee JK, Kwon JH (2007) On the computation and approximation of ultra-highdegree spherical harmonic series. J Geod 81(9): 603-615. doi: 10.1007/s00190-0060123-z

Keller W, Sharifi MA (2005) Satellite gradiometry using a satellite pair. J Geod 78(9):544557, doi: 10.1007/s00190-004-0426-X

Marti U (2004) High-precision combined geoid determination in Switzerland. Presented at Gravity, Geoid and Space Missions (GGSM) 2004 Symposium, Porto, Aug 30 - Sep 03 2004, Porto, Portugal

Marti U, Hirt C, Schlatter A (2011) Local Realisation of a World Height System by Using Levelling Data. Presented at IUGG General Assembly 2011, Melbourne, Australia, Paper \# 3634

Meyer TH, Roman DR, Zilkoski DB (2006) What does height really mean? Part III: Height Systems. Surveying and Land Information Science 66(2):149-160

Nahavandchi H (2002) Two different methods of geoidal height determinations using a spherical harmonic representation of the geopotential, topographic corrections and the height anomaly-geoidal height difference. J Geod 76(6-7):345-352. doi 10.1007/s00190-002-0253-X

Pavlis NK, Factor JK, Holmes SA (2007) Terrain-related gravimetric quantities computed for the next EGM. Proceedings of the 1st International Symposium of the International Gravity Field Service (IGFS), Istanbul, pp 318-323

Pavlis NK, Holmes SA, Kenyon SC, Factor JK (2008) An Earth Gravitational Model to Degree 2160: EGM2008. Presented at the 2008 General Assembly of the European Geoscience Union, Vienna, Austria, April 13-18, 2008

Rapp RH (1997) Use of potential coefficient models for geoid undulation determinations using a spherical harmonic representation of the height anomaly/geoid undulation difference. J Geod, 71(5), 282-289

Rizos C (1979) An efficient computer technique for the evaluation of geopotential from spherical harmonic models. Aust J Geod Photogram Surv 31: 161-169

Rummel R, van Gelderen M (1995) Meissl scheme - spectral characteristics of physical geodesy. Manuscr Geod 20(5): 379-385

Sjöberg LE (2006) A refined conversion from normal height to orthometric height. Stud Geophys Geod 50(4): 595-606. doi: 10.1007/s11200-006-0037-5 
Sjöberg LE (2010) A strict formula for geoid-to-quasigeoid separation. J Geod 84(11):699702. doi: 10.1007/s00190-010-0407-1

Smith DA (1998) There is no such thing as "The" EGM96 geoid: Subtle points on the use of a global geopotential model. In: IGeS Bulletin No. 8, International Geoid Service, Milan, Italy, 17-28

Šprlák M (2011) On the numerical problems of spherical harmonics: Numerical and algebraic methods avoiding instabilities of Associated Legendre's Functions. Zeitschrift für Geodäsie, Geoinformation und Landmanagement (zfv) 136(5): 310-320

Tenzer R, Novák P, Moore P, Kuhn M, Vaníček P (2006) Explicit formula for the geoidquasigeoid separation. Stud Geophys Geod 50(4): 607-618. doi: 10.1007/s11200-0060038-4

Tóth G (2005) The gradiometric-geodynamic boundary value problem. In: Jekeli C,Bastos L, Fernandes J (eds) Gravity,Geoid and Space Missions, International Association of Geodesy Symposia 129, Springer, Berlin, pp 352-257

Tscherning CC, Poder K (1982) Some geodetic applications of Clenshaw summation. Boll Geofis Sci Aff 4: 351-364

Tscherning CC, Rapp RH, Goad C (1983) A comparison of methods for computing gravimetric quantities from high degree spherical harmonic expansions. Manuscr Geod 8: $249-272$

Torge W (2001) Geodesy, third edition, De Gruyter, Berlin New York

Wenzel H-G (1985) Hochauflösende Kugelfunktionsmodelle für das Gravitationspotential der Erde. Wiss. Arb. Fachr. Vermessungswesen Universität Hannover $\mathrm{Nr}$ 137, Hannover, Germany

Wenzel H-G (1998) Ultra high degree geopotential models GPM98A and GPM98B to degree 1,800. In: Proceedings of joint meeting international gravity commission and international geoid commission, Budapest, 10-14 March, Finnish Geodetic Institute, Helsinki, Report 98/4, pp 71-80

Wenzel H-G (1999) Schwerefeldmodellierung durch ultra hochauflösende Kugelfunktionsmodelle. Zeitschrift für Vermessungswesen 124(5):144-154

Wessel P, Smith WHF (1998) New, improved version of the Generic Mapping Tools released. EOS Trans. AGU 79, 579

Wieczorek MA (2007) Gravity and topography of the terrestrial planets. In: Treatise on Geophysics, 10, 165-206. Elsevier-Pergamon, Oxford

Wittwer T, Klees R, Seitz K, Heck B (2008) Ultra-high degree spherical harmonic analysis and synthesis using extended-range arithmetic. J Geod 82(4-5):223-229. doi 10.1007/s00190-007-0172-y 